\title{
Kink-Like Wave and Compacton-Like Wave Solutions for a Two-Component Fornberg-Whitham Equation
}

\author{
Shaoyong $\mathrm{Li}^{1}$ and Ming Song ${ }^{2,3}$ \\ ${ }^{1}$ School of Mathematics and Information Sciences, Shaoguan University, Shaoguan, Guangdong 512005, China \\ ${ }^{2}$ Department of Mathematics, Shaoxing University, Shaoxing, Zhejiang 312000, China \\ ${ }^{3}$ Department of Mathematics, Faculty of Sciences, Yuxi Normal University, Yuxi, Yunnan 653100, China
}

Correspondence should be addressed to Shaoyong Li; lishaoyongok@sohu.com

Received 28 December 2013; Revised 19 January 2014; Accepted 23 January 2014; Published 16 March 2014

Academic Editor: Massimo Furi

Copyright (C) 2014 S. Li and M. Song. This is an open access article distributed under the Creative Commons Attribution License, which permits unrestricted use, distribution, and reproduction in any medium, provided the original work is properly cited.

Using bifurcation method and numerical simulation approach of dynamical systems, we study a two-component FornbergWhitham equation. Two types of bounded traveling wave solutions are found, that is, the kink-like wave and compacton-like wave solutions. The planar graphs of these solutions are simulated by using software Mathematica; meanwhile, two new phenomena are revealed; that is, the periodic wave solution can become the kink-like wave or compacton-like wave solution under some conditions, respectively. Exact implicit or parameter expressions of these solutions are given.

\section{Introduction}

The Fornberg-Whitham equation

$$
u_{t}-u_{x x t}+u_{x}+u u_{x}=u u_{x x x}+3 u_{x} u_{x x}
$$

was used to study the qualitative behaviors of wave breaking [1]. It admits a wave of the greatest height, as a peaked limiting form of the traveling wave solution [2], $u(x, t)=$ $\widetilde{A} \exp ((1 / 2)|x-(4 / 3) t|)$, where $\widetilde{A}$ is an arbitrary constant. Recently, Zhou and Tian found that (1) possess kink-like wave solutions in [3]. They obtained some solitons, peakons, and periodic cusp wave solutions in [4]. Further, they obtained the smooth periodic wave solutions and loopsoliton solutions by using elliptic integral [5]. Feng and $\mathrm{Wu}$ [6] considered the classification of single traveling wave solutions to (1). Chen et al. [7] gave some smooth periodic wave, smooth solitary wave, periodic cusp wave, and loopsoliton solutions of (1) and made the numerical simulation.

He et al. [8] studied the following modified FornbergWhitham equation:

$$
u_{t}-u_{x x t}+u_{x}+u^{2} u_{x}=u u_{x x x}+3 u_{x} u_{x x} .
$$

In some parametric conditions, some peakons and solitary waves were found and their exact parametric representations in explicit form were obtained.

Jiang and $\mathrm{Bi}$ [9] considered the Fornberg-Whitham equation with linear dispersion term given by

$$
u_{t}-u_{x x t}+u_{x}+u u_{x}=u u_{x x x}+3 u_{x} u_{x x}-\gamma u_{x x x}
$$

where $\gamma$ is a real constant. When $\gamma=0$, (3) reduces to (1). They investigated the existence of the smooth and nonsmooth traveling wave solutions and gave some analytic expressions of smooth solitary wave, periodic cusp wave, and peakon solutions for (3).

Fan et al. [10] presented a two-component FornbergWhitham equation given by

$$
\begin{gathered}
u_{t}=u_{x x t}-u_{x}-u u_{x}+u u_{x x x}+3 u_{x} u_{x x}+\rho_{x} \\
\rho_{t}=-(\rho u)_{x}
\end{gathered}
$$

where $u=u(x, t)$ is the height of the water surface above a horizontal bottom and $\rho=\rho(x, t)$ is related to the horizontal velocity field. When $\rho=0$, (4) reduces to (1). Parametric conditions to smooth soliton solution, 
kink solution, antikink solution, and uncountable infinite many smooth periodic wave solutions of (4) were given. Later, Wen [11] further studied (4). He presented all possible phase portraits determinately and gave all the exact explicit parametric conditions for various solutions.

The concept of compacton: soliton with compact support or strict localization of solitary waves appeared in the work of Rosenau and Hyman [12], where a genuinely nonlinear dispersive equation $K(n, n)$ defined by

$$
u_{t}+a\left(u^{n}\right)_{x}+\left(u^{n}\right)_{x x x}=0
$$

was subjected to experimental and analytical studies. They found certain solitary wave solutions which vanish identically outside a finite core region. These solutions are called compactons. Several studies have been conducted in [13-18]. The kink-like wave or generalized kink wave is discovered by Liu et al. [14], which is defined on semifinal bounded domain and possesses some properties of the kink wave.

Many methods have been used to investigate traveling wave solutions to nonlinear equations, such as Jacobi elliptic function method $[19,20]$, F-expansion and extended Fexpansion method $[21,22]$, and $\left(G^{\prime} / G\right)$-expansion method $[23,24]$. Here, our aim in this paper is to use the bifurcation method of dynamical systems [25-28] to investigate (4). We obtain the kink-like wave and compacton-like wave solutions with implicit or parameter expressions. The planar graphs of these solutions are simulated by using software Mathematica; meanwhile, we point out that the periodic wave solution can become the kink-like wave or compacton-like wave solution under some conditions, respectively. To the best of our knowledge, these solutions and phenomena are new for (4). Our work may help people to know deeply the described physical process and possible applications of (4).

The remainder of this paper is organized as follows. In Section 2, we study the bifurcation phase portraits. In Section 3, we make the numerical simulation for bounded integral curves. In Section 4, we derive the exact implicit or parameter expressions of the kink-like wave and compactonlike wave solutions. A brief conclusion is given in Section 5.

\section{Bifurcation Phase Portraits}

We look for the traveling wave solutions of (4) in the form of

$$
u(x, t)=\varphi(\xi)+\widetilde{c}, \quad \rho(x, t)=\psi(\xi), \quad \xi=x-c t,
$$

where $\widetilde{c}$ is the mean level and $c$ is the wave speed.

Substituting (6) into (4) and integrating once with respect to $\xi$, it follows that

$$
\begin{gathered}
(c-\widetilde{c}-1) \varphi-\frac{1}{2} \varphi^{2}+\left(\varphi^{\prime}\right)^{2}+(\varphi+\widetilde{c}-c) \varphi^{\prime \prime}+\psi=\theta, \\
\psi=-\frac{g}{\varphi+\widetilde{c}-c},
\end{gathered}
$$

where $\theta, g$ are two integral constants and $g \neq 0$ (if $g=0$, then $\rho=0$ from the second equation of (7) and (6). In this case (4) reduces to (1), which was studied in [3-7]).
In order to study conveniently, we choose $\widetilde{c}=c$, and this only makes a translational movement of the singular line from $\varphi=c-\widetilde{c}$ to $\varphi=0$, so there is no essential difference for the results. Thus, substituting the second equation of (7) into the first equation of (7), we obtain

$$
\varphi^{2} \varphi^{\prime \prime}=\frac{1}{2} \varphi^{3}+\varphi^{2}+\theta \varphi+g-\varphi\left(\varphi^{\prime}\right)^{2} .
$$

Letting $y=\varphi^{\prime}$, we obtain the following planar system:

$$
\begin{gathered}
\frac{\mathrm{d} \varphi}{\mathrm{d} \xi}=y, \\
\frac{\mathrm{d} y}{\mathrm{~d} \xi}=\frac{(1 / 2) \varphi^{3}+\varphi^{2}+\theta \varphi+g-\varphi y^{2}}{\varphi^{2}},
\end{gathered}
$$

under the transformation $d \xi=\varphi^{2} d \tau$, and system (9) becomes

$$
\begin{gathered}
\frac{\mathrm{d} \varphi}{\mathrm{d} \tau}=y \varphi^{2}, \\
\frac{\mathrm{d} y}{\mathrm{~d} \tau}=\frac{1}{2} \varphi^{3}+\varphi^{2}+\theta \varphi+g-\varphi y^{2} .
\end{gathered}
$$

Obviously, system (9) and system (10) have the same first integral

$$
H(\varphi, y)=\varphi^{2} y^{2}-\left(\frac{1}{4} \varphi^{4}+\frac{2}{3} \varphi^{3}+\theta \varphi^{2}+2 g \varphi\right)=h,
$$

where $h$ is an integral constant. Consequently, these two systems have the same topological phase portraits except for the straight line $\varphi=0$. Thus, we can understand the phase portraits of system (9) from those of system (10).

In order to state conveniently, for given constants $\theta$ and $g$, let

$$
\begin{gathered}
f_{0}(\varphi)=\frac{1}{2} \varphi^{3}+\varphi^{2}+\theta \varphi+g, \\
g_{1}(\theta)=\frac{2}{27}(-2(2+\sqrt{4-6 \theta})+3(3+\sqrt{4-6 \theta}) \theta), \\
g_{2}(\theta)=-\frac{2}{729}(4+\sqrt{16-27 \theta})(8+2 \sqrt{16-27 \theta}-27 \theta), \\
g_{3}(\theta)=-\frac{2}{729}(-4+\sqrt{16-27 \theta})(-8+2 \sqrt{16-27 \theta}+27 \theta), \\
g_{4}(\theta)=-\frac{2}{27}(4-2 \sqrt{4-6 \theta}+3(-3+\sqrt{4-6 \theta}) \theta), \\
g_{5}(\theta)=\frac{2}{27}(-4+9 \theta) .
\end{gathered}
$$

Assume that $\varphi_{1}, \varphi_{2}$, and $\varphi_{3}$ are three roots of equation $f_{0}(\varphi)=0$, where

$$
\begin{aligned}
& \varphi_{1}=-\frac{2}{3}+\frac{1}{6}(-1+\sqrt{3} i) \delta+\frac{(1+\sqrt{3} i)(3 \theta-2)}{3 \delta}, \\
& \varphi_{2}=-\frac{2}{3}-\frac{1}{6}(1+\sqrt{3} i) \delta+\frac{(1-\sqrt{3} i)(3 \theta-2)}{3 \delta},
\end{aligned}
$$


TABLE 1: The singular point and phase portrait for different cases.

\begin{tabular}{|c|c|c|c|c|c|}
\hline Case & Root of $f_{0}(\varphi)=0$ & Center & Saddle & Degenerate saddle & Phase portrait \\
\hline Case 1 & $\varphi_{3}>0$ & - & $\left(\varphi_{3}, 0\right)$ & - & Figure 1(a) \\
\hline Case 2 & $\varphi_{1}=\varphi_{2}<0<\varphi_{3}$ & - & $\left(\varphi_{3}, 0\right)$ & $\left(\varphi_{1}, 0\right)$ & Figure 1(b) \\
\hline Case 3 & $\varphi_{1}<\varphi_{2}<0<\varphi_{3}$ & $\left(\varphi_{2}, 0\right)$ & $\left(\varphi_{1}, 0\right)\left(\varphi_{3}, 0\right)$ & - & Figure 1(c) \\
\hline Case 4 & $\varphi_{1}<\varphi_{2}<0<\varphi_{3}$ & $\left(\varphi_{2}, 0\right)$ & $\left(\varphi_{1}, 0\right)\left(\varphi_{3}, 0\right) H\left(\varphi_{1}, 0\right)=0$ & - & Figure $1(d)$ \\
\hline Case 5 & $\varphi_{1}<\varphi_{2}<0<\varphi_{3}$ & $\left(\varphi_{2}, 0\right)$ & $\left(\varphi_{1}, 0\right)\left(\varphi_{3}, 0\right)$ & - & Figure 1(e) \\
\hline Case 6 & $\varphi_{1}<0<\varphi_{2}<\varphi_{3}$ & $\left(\varphi_{2}, 0\right)$ & $\left(\varphi_{1}, 0\right)\left(\varphi_{3}, 0\right)$ & - & Figure 1(f) \\
\hline Case 7 & $\varphi_{1}<0<\varphi_{2}<\varphi_{3}$ & $\left(\varphi_{2}, 0\right)$ & $\left(\varphi_{1}, 0\right)\left(\varphi_{3}, 0\right) H\left(\varphi_{3}, 0\right)=0$ & - & Figure $1(\mathrm{~g})$ \\
\hline Case 8 & $\varphi_{1}<0<\varphi_{2}<\varphi_{3}$ & $\left(\varphi_{2}, 0\right)$ & $\left(\varphi_{1}, 0\right)\left(\varphi_{3}, 0\right)$ & - & Figure $1(\mathrm{~h})$ \\
\hline Case 9 & $\varphi_{1}<0<\varphi_{2}=\varphi_{3}$ & - & $\left(\varphi_{1}, 0\right)$ & $\left(\varphi_{3}, 0\right)$ & Figure 1(i) \\
\hline Case 10 & $\varphi_{1}<0$ & - & $\left(\varphi_{1}, 0\right)$ & - & Figure 1(j) \\
\hline Case 11 & $\varphi_{1}<\varphi_{2}<\varphi_{3}<0$ & $\left(\varphi_{2}, 0\right)$ & $\left(\varphi_{1}, 0\right)\left(\varphi_{3}, 0\right)$ & - & Figure $1(\mathrm{k})$ \\
\hline Case 12 & $\varphi_{1}<\varphi_{2}=\varphi_{3}<0$ & - & $\left(\varphi_{1}, 0\right)$ & $\left(\varphi_{2}, 0\right)$ & Figure 1(l) \\
\hline Case 13 & $\varphi_{1}<\varphi_{2}<\varphi_{3}<0$ & $\left(\varphi_{2}, 0\right)$ & $\left(\varphi_{1}, 0\right)\left(\varphi_{3}, 0\right)$ & - & Figure $1(\mathrm{~m})$ \\
\hline Case 14 & $\varphi_{1}<\varphi_{2}<\varphi_{3}<0$ & $\left(\varphi_{2}, 0\right)$ & $\left(\varphi_{1}, 0\right)\left(\varphi_{3}, 0\right) H\left(\varphi_{1}, 0\right)=H\left(\varphi_{3}, 0\right)$ & - & Figure $1(\mathrm{n})$ \\
\hline Case 15 & $\varphi_{1}=\varphi_{2}<\varphi_{3}<0$ & - & $\left(\varphi_{3}, 0\right)$ & $\left(\varphi_{1}, 0\right)$ & Figure $1(\mathrm{o})$ \\
\hline Case 16 & $\varphi_{1}<\varphi_{2}<\varphi_{3}<0$ & $\left(\varphi_{2}, 0\right)$ & $\left(\varphi_{1}, 0\right)\left(\varphi_{3}, 0\right)$ & - & Figure $1(\mathrm{p})$ \\
\hline
\end{tabular}

$$
\begin{gathered}
\varphi_{3}=-\frac{2}{3}+\frac{4-6 \theta}{3 \delta}+\frac{\delta}{3} \\
\delta=(-8-27 g+18 \theta \\
\left.+3 \sqrt{3} \sqrt{g(16+27 g)-36 g \theta-4 \theta^{2}+8 \theta^{3}}\right)^{1 / 3}
\end{gathered}
$$

Meanwhile, we give conditions as follows.

Case 1. $\theta<(2 / 3), g<g_{1}(\theta)$ or $\theta \geq(2 / 3), g<0$.

Case 2. $\theta<(2 / 3)(\theta \neq(1 / 2))$ and $g=g_{1}(\theta)$.

Case 3. $\theta<(1 / 2)$ and $g_{1}(\theta)<g<g_{2}(\theta)$.

Case 4. $\theta<(1 / 2)$ and $g=g_{2}(\theta)$.

Case 5. $\theta<(1 / 2)$ and $g_{2}(\theta)<g<0$.

Case 6. $\theta \leq 0$ and $0<g<g_{3}(\theta)$.

Case 7. $\theta \leq 0$ and $g=g_{3}(\theta)$.

Case 8. $\theta \leq 0$ and $g_{3}(\theta)<g<g_{4}(\theta)$.

Case 9. $\theta \leq 0$ and $g=g_{4}(\theta)$.

Case 10. $\theta<(2 / 3), g>g_{4}(\theta)$ or $\theta \geq(2 / 3), g>0$.

Case 11. $0<\theta<(1 / 2), 0<g<g_{4}(\theta)$ or $(1 / 2) \leq \theta<(2 / 3)$, $g_{5}(\theta)<g<g_{4}(\theta)$.

Case 12. $0<\theta<(2 / 3)$ and $g=g_{4}(\theta)$.

Case 13. $\theta=(1 / 2)$ and $0<g<g_{5}(\theta)$.
Case 14. $(1 / 2) \leq \theta<(2 / 3)$ and $g=g_{5}(\theta)$.

Case 15. $(1 / 2)<\theta<(2 / 3)$ and $g=g_{1}(\theta)$.

Case 16. $(1 / 2)<\theta<(2 / 3)$ and $g_{1}(\theta)<g<g_{5}(\theta)$.

According to the qualitative theory of differential equations and the above conditions, we have the results as Table 1.

\section{Numerical Simulation for Bounded Integral Curves}

In this section, we make the numerical simulation for bounded integral curves. For convenience, throughout the following work we only discuss the solution $\varphi(\xi)$ with respect to the first component $u=\varphi(\xi)+c$ and omit the second component $\rho=\psi(\xi)=-g / \varphi$ of $(4)$.

From the derivation in Section 2 we see that the bounded traveling waves of (4) correspond to the bounded integral curves of (8) and the bounded integral curves of (8) correspond to the orbits of system (9) in which $\varphi=\varphi(\xi)$ is bounded. Therefore we can simulate the bounded integral curves of (8) by using the information of the phase portraits of system (9).

It follows from [14-18] that the open orbits $K_{i}(i=1-5)$ of system (9) correspond to the compacton-like waves of (4), the heteroclinic orbits $J_{i}(i=1,2)$ of system (9) correspond to the kink-like waves of (4), and the periodic orbits surrounding the center point $\left(\varphi_{2}, 0\right)$ correspond to the periodic waves of (4). Here we only make the numerical simulation for Cases 4 and 5 as Examples 1 and 2. The other cases are similar to Examples 1 and 2, so we omit them.

Example 1. For Case 4, taking $\theta=-1<1 / 2$, then $g=g_{2}(\theta)=$ $-1.39361, \varphi_{1}=-2.3461, \varphi_{2}=-0.930567$, and $\varphi_{3}=1.27666$. 
(1) From (11), the two heteroclinic orbits $J_{1}^{ \pm}$(see Figure $1(d))$ passing through the saddle point $\left(\varphi_{3}, 0\right)$ have expressions, respectively,

$$
y= \pm \sqrt{\frac{(1 / 4) \varphi^{4}+(2 / 3) \varphi^{3}+\theta \varphi^{2}+2 g \varphi+h\left(\varphi_{3}\right)}{\varphi^{2}}},
$$

where $h\left(\varphi_{3}\right)=H\left(\varphi_{3}, 0\right)$ and $0<\varphi<\varphi_{3}$. We assume that $\varphi(0)$ and $\varphi^{\prime}(0)$ are the initial values for the orbit of system (9). For any given $\varphi_{1}^{*}\left(0<\varphi_{1}^{*}<\varphi_{3}\right)$, then from the first equation of system (9) we have $\varphi^{\prime}=y\left(\varphi_{1}^{*}\right)$ at $\varphi=\varphi_{1}^{*}$. For example, setting $\varphi_{1}^{*}=1$, we have $y\left(\varphi_{1}^{*}\right)= \pm 0.5160826789900144$. Thus taking $\varphi(0)=1$ and $\varphi^{\prime}(0)= \pm 0.5160826789900144$ as initial values, respectively, we simulate the integral curves of (8) as (a) and (b) in Figure 2.

(2) From (11), the two heteroclinic orbits $J_{2}^{ \pm}$(see Figure $1(\mathrm{~d}))$ passing through the saddle point $\left(\varphi_{1}, 0\right)$ have expressions, respectively,

$$
y= \pm \sqrt{\frac{(1 / 4) \varphi^{4}+(2 / 3) \varphi^{3}+\theta \varphi^{2}+2 g \varphi+h\left(\varphi_{1}\right)}{\varphi^{2}}},
$$

where $h\left(\varphi_{1}\right)=H\left(\varphi_{1}, 0\right)=H(0,0)=0$ and $\varphi_{1}<\varphi<0$. For any given $\varphi_{2}^{*}\left(\varphi_{1}<\varphi_{2}^{*}<0\right)$, then from the first equation of system (9) we have $\varphi^{\prime}=y\left(\varphi_{2}^{*}\right)$ at $\varphi=\varphi_{2}^{*}$. For example, setting $\varphi_{2}^{*}=-1$, we have $y\left(\varphi_{2}^{*}\right)= \pm 1.1707040231039854$. Thus taking $\varphi(0)=-1$ and $\varphi^{\prime}(0)= \pm 1.1707040231039854$ as initial values, respectively, we simulate the integral curves of (8) as (c) and (d) in Figure 2.

(3) From (11), the orbit $K_{1}$ (see Figure 1(d)) passing through $\left(\varphi_{0}, 0\right)$ has expressions

$$
y= \pm \sqrt{\frac{(1 / 4) \varphi^{4}+(2 / 3) \varphi^{3}+\theta \varphi^{2}+2 g \varphi+h\left(\varphi_{0}\right)}{\varphi^{2}}}
$$

where $h\left(\varphi_{0}\right)=H\left(\varphi_{0}, 0\right), 0<\varphi \leq \varphi_{0}$, and $0<\varphi_{0}<\varphi_{3}$. Choosing $\varphi_{0}=1 \in\left(0, \varphi_{3}\right)$ and taking $\varphi(0)=\varphi_{0}$ and $\varphi^{\prime}(0)=0$ as initial values, respectively, we simulate the integral curve of (8) as (e) in Figure 2.

Example 2. For Case 5, taking $\theta=-1<(1 / 2)$, then $g=$ $(1 / 2) g_{2}(\theta)=-0.696804 \in\left(g_{2}(\theta), 0\right), \varphi_{1}=-2.56755, \varphi_{2}=$ -0.50572 , and $\varphi_{3}=1.07327$. From (11), the orbit $K_{3}$ (see Figure $1(\mathrm{e}))$ passing through $\left(\varphi_{3 r}, 0\right)$ has expressions

$$
y= \pm \sqrt{\frac{(1 / 4) \varphi^{4}+(2 / 3) \varphi^{3}+\theta \varphi^{2}+2 g \varphi+h\left(\varphi_{3}\right)}{\varphi^{2}}},
$$

where $h\left(\varphi_{3}\right)=H\left(\varphi_{3}, 0\right), \varphi_{3 r} \leq \varphi<0$, and $\varphi_{3 r}=$ -1.624378546433569 . Taking $\varphi(0)=\varphi_{3 r}$ and $\varphi^{\prime}(0)=0$ as initial values, respectively, we simulate the integral curve of (8) as (f) in Figure 2.

Remark 3. The kink-like waves in Figures 2(a) and 2(b) are defined on $\left(-\infty, \widetilde{\xi}_{1}\right)$ and $\left(-\widetilde{\xi}_{1},+\infty\right)$, respectively. The kink-like waves in Figures 2(c) and 2(d) are defined on $\left(-\widetilde{\xi}_{2},+\infty\right)$ and $\left(-\infty, \widetilde{\xi}_{2}\right)$, respectively. The compacton-like wave in Figure $2(\mathrm{e})$ has peak form on $\left(-\widetilde{\xi}_{3}, \widetilde{\xi}_{3}\right)$, where $\widetilde{\xi}_{1}, \widetilde{\xi}_{2}$, and $\widetilde{\xi}_{3}$ satisfy

$$
\begin{aligned}
& \tilde{\xi}_{1}=\int_{0}^{\varphi_{1}^{*}} \frac{s \mathrm{~d} s}{\sqrt{(1 / 4) s^{4}+(2 / 3) s^{3}+\theta s^{2}+2 g s+h\left(\varphi_{3}\right)}}, \quad \text { for } 0<\varphi_{1}^{*}<\varphi_{3}, \\
& \tilde{\xi}_{2}=\int_{\varphi_{2}^{*}}^{0}, \quad \text { for } \varphi_{1}<\varphi_{2}^{*}<0, \\
& \tilde{\xi}_{3}=\int_{0}^{\varphi_{0}} \frac{-s \mathrm{~d} s}{\sqrt{(1 / 4) s^{4}+(2 / 3) s^{3}+\theta s^{2}+2 g s+h\left(\varphi_{1}\right)}}
\end{aligned}
$$

where $h\left(\varphi_{0}\right)=H\left(\varphi_{0}, 0\right), h\left(\varphi_{1}\right)=H\left(\varphi_{1}, 0\right)$, and $h\left(\varphi_{3}\right)=$ $H\left(\varphi_{3}, 0\right)$. Take the data of Example 1, that is, $\theta=-1, g=$ $-1.39361, \varphi_{1}^{*}=1, \varphi_{2}^{*}=-1, \varphi_{3}=1.27666, \varphi_{1}=-2.3461$, and $\varphi_{0}=1$, then from (18) we obtain $\widetilde{\xi}_{1}=0.54746, \widetilde{\xi}_{2}=0.480057$, and $\widetilde{\xi}_{3}=0.915546$ which are identical with the simulations (see Figures 2(a)-2(e)).

Remark 4. The compacton-like wave in Figure 2(f) is defined on $\left(-\widetilde{\xi}_{4}, \widetilde{\xi}_{4}\right)$, where $\widetilde{\xi}_{4}$ satisfies

$$
\tilde{\xi}_{4}=\int_{\varphi_{3 r}}^{0} \frac{-s \mathrm{~d} s}{\sqrt{(1 / 4) s^{4}+(2 / 3) s^{3}+\theta s^{2}+2 g s+h\left(\varphi_{3}\right)}}
$$

where $h\left(\varphi_{3}\right)=H\left(\varphi_{3}, 0\right)$. Take the data of Example 2; that is, $\theta=-1, g=-0.696804, \varphi_{3}=1.07327$, and $\varphi_{3 r}=-1.624378546433569$, and then from (19) we obtain $\widetilde{\xi}_{4}=1.73392$ which is identical with the simulation (see Figure 2(f)).

Remark 5. For Cases 4 and 5, there are a family of periodic orbits surrounding the center point $\left(\varphi_{2}, 0\right)$, but the boundaries of the periodic orbits are different. For Case 4 , the boundaries of the periodic orbits are the two heteroclinic orbits $J_{2}^{ \pm}$(see Figure 3(a)), while for Case 5, the boundary of the periodic orbits is the open orbit $K_{4}$ (see Figure 3(b)). Taking the data of Example 1 and a set of initial values $\left(\varphi(0), \varphi^{\prime}(0)\right)$, that is, $\theta=-1, g=-1.39361$ and $\left(\varphi(0), \varphi^{\prime}(0)\right)=(-1,0.7),(-1,1.1),(-1,1.17),(-1,1.170704)$, we simulate the periodic orbits of (8) as Figure 4. Similarly, taking the data of Example 2 and a set of initial values $\left(\varphi(0), \varphi^{\prime}(0)\right)$, that is, $\theta=-1, g=-0.696804$, and $\left(\varphi(0), \varphi^{\prime}(0)\right)=(-0.7,0),(-0.9,0),(-0.98,0),(-0.9854465,0)$, we simulate the periodic orbits of (8) as Figure 5. The simulations in Figure 4 imply that the periodic waves tend to two kink-like waves when the periodic orbits tend to the heteroclinic orbits $J_{2}^{ \pm}$. The simulations in Figure 5 imply that the periodic waves tend to the periodic compacton-like wave when the periodic orbits tend to the open orbit $K_{4}$. 


\section{The Expressions of Kink-Like and Compacton-Like Waves}

In this section we derive the exact expressions of the kinklike and compacton-like waves in different cases $i(i=1-16)$. Assuming that $\left(\varphi(0), \varphi^{\prime}(0)\right)$ is the initial point of an orbit of system (9). Let

$$
F(\varphi)=\frac{1}{4} \varphi^{4}+\frac{2}{3} \varphi^{3}+\theta \varphi^{2}+2 g \varphi+h_{0}
$$

where $h_{0}=H\left(\varphi(0), \varphi^{\prime}(0)\right)$, then form $H(\varphi, y)=h_{0}$, the following equation:

$$
\varphi^{2} y^{2}=F(\varphi)
$$

determines the orbit passing through $\left(\varphi(0), \varphi^{\prime}(0)\right)$.

4.1. Solutions of Kink-Like Wave. (1) In Cases $i(i=1-4)$ corresponding to phase portraits in Figures 1(a)-1(d), (20) becomes

$$
F(\varphi)=\frac{1}{4}\left(\varphi_{3}-\varphi\right)^{2}\left(\varphi^{2}+m_{1} \varphi+n_{1}\right)
$$

where $m_{1}=(8 / 3)+2 \varphi_{3}, n_{1}=4 \theta+(1 / 3) \varphi_{3}\left(16+9 \varphi_{3}\right)$. Thus the orbits $J_{1}^{ \pm}$passing through saddle point $\left(\varphi_{3}, 0\right)$ have expressions

$$
y= \pm \frac{\left(\varphi_{3}-\varphi\right) \sqrt{\varphi^{2}+m_{1} \varphi+n_{1}}}{2 \varphi}
$$

where $0<\varphi<\varphi_{3}$. Substituting (23) into $\mathrm{d} \varphi / \mathrm{d} \xi=y$ and integrating along $J_{1}^{+}$and $J_{1}^{-}$for initial value $\varphi(0)=\varphi_{1}^{*}$, where $0<\varphi_{1}^{*}<\varphi_{3}$, we obtain two kink-like wave solutions of implicit expression as follows:

$$
\begin{array}{ll}
f_{1}(\varphi)=f_{1}\left(\varphi_{1}^{*}\right) e^{(1 / 2) \xi}, & -\xi_{1}<\xi<\infty, \\
f_{1}(\varphi)=f_{1}\left(\varphi_{1}^{*}\right) e^{-(1 / 2) \xi}, & -\infty<\xi<\xi_{1},
\end{array}
$$

where

$$
\begin{gathered}
\xi_{1}=2 \ln \frac{f_{1}\left(\varphi_{1}^{*}\right)}{f_{1}(0)}, \\
f_{1}(s)=\frac{\left(2 \sqrt{a_{1}} \sqrt{s^{2}+m_{1} s+n_{1}}+b_{1}\left(\varphi_{3}-s\right)+2 a_{1}\right)^{\mu_{1}}}{\left(\varphi_{3}-s\right)^{\mu_{1}}\left(2 \sqrt{s^{2}+m_{1} s+n_{1}}+2 s+m_{1}\right)}, \\
\mu_{1}=\frac{\varphi_{3}}{\sqrt{a_{1}}}, \\
a_{1}=\varphi_{3}^{2}+m_{1} \varphi_{3}+n_{1}, \quad b_{1}=-m_{1}-2 \varphi_{3} .
\end{gathered}
$$

The derivations of other kink-like wave solutions are similar to the above case, so we omit the details and only list the results.
(2) In Cases $i(i=5,6)$ corresponding to phase portraits in Figures 1(e) and 1(f), we obtain two kink-like wave solutions of implicit expression as follows:

$$
\begin{array}{ll}
f_{2}(\varphi)=f_{2}\left(\varphi_{1}^{*}\right) e^{(1 / 2) \xi}, & -\xi_{2}<\xi<\infty, \\
f_{2}(\varphi)=f_{2}\left(\varphi_{1}^{*}\right) e^{-(1 / 2) \xi}, & -\infty<\xi<\xi_{2},
\end{array}
$$

where

$$
\begin{gathered}
\xi_{2}=2 \ln \frac{f_{2}\left(\varphi_{1}^{*}\right)}{f_{2}(0)}, \\
f_{2}(s)=\frac{\left(2 \sqrt{a_{2}} \sqrt{\left(s-\varphi_{3 l}\right)\left(s-\varphi_{3 r}\right)}+b_{2}\left(\varphi_{3}-s\right)+2 a_{2}\right)^{\mu_{2}}}{\left(\varphi_{3}-s\right)^{\mu_{2}}\left(2 \sqrt{\left(s-\varphi_{3 l}\right)\left(s-\varphi_{3 r}\right)}+2 s-\varphi_{3 l}-\varphi_{3 r}\right)}, \\
\mu_{2}=\frac{\varphi_{3}}{\sqrt{a_{2}}}, \\
\varphi_{3 l}=\frac{1}{3}\left(-4-3 \varphi_{3}-\sqrt{2} \sqrt{8-18 \theta-3 \varphi_{3}\left(4+3 \varphi_{3}\right)}\right), \\
\varphi_{3 r}=\frac{1}{3}\left(-4-3 \varphi_{3}+\sqrt{2} \sqrt{8-18 \theta-3 \varphi_{3}\left(4+3 \varphi_{3}\right)}\right) .
\end{gathered}
$$

(3) In Case 7 corresponding to phase portrait in Figure 1(g), we obtain two kink-like wave solutions of implicit expression as follows:

$$
\begin{array}{ll}
f_{3}(\varphi)=f_{3}\left(\varphi_{1}^{*}\right) e^{(1 / 2) \xi}, & -\xi_{3}<\xi<\infty, \\
f_{3}(\varphi)=f_{3}\left(\varphi_{1}^{*}\right) e^{-(1 / 2) \xi}, & -\infty<\xi<\xi_{3},
\end{array}
$$

where

$$
\begin{gathered}
\xi_{3}=2 \ln \frac{f_{3}\left(\varphi_{1}^{*}\right)}{f_{3}(0)}, \\
f_{3}(s)=\frac{\left(\sqrt{s-\varphi_{3}^{\circ}}-\sqrt{s}\right)\left(\sqrt{\varphi_{3}\left(s-\varphi_{3}^{\circ}\right)}+\sqrt{\left(\varphi_{3}-\varphi_{3}^{\circ}\right) s}\right)^{\mu_{3}}}{\left(\sqrt{s-\varphi_{3}^{\circ}}+\sqrt{s}\right)\left(\sqrt{\varphi_{3}\left(s-\varphi_{3}^{\circ}\right)}-\sqrt{\left(\varphi_{3}-\varphi_{3}^{\circ}\right)}\right)^{\mu_{3}}}, \\
\mu_{3}=\sqrt{\frac{\varphi_{3}}{\varphi_{3}-\varphi_{3}^{\circ}}}, \quad \varphi_{3}^{\circ}=-\frac{8}{3}-2 \varphi_{3} .
\end{gathered}
$$

(4) In Case 11 corresponding to phase portraits in Figure 1(k), we obtain two kink-like wave solutions of implicit expression as follows:

$$
\begin{array}{ll}
f_{4}(\varphi)=f_{4}\left(\varphi_{1}^{*}\right) e^{(1 / 2) \xi}, & -\infty<\xi<\xi_{4}, \\
f_{4}(\varphi)=f_{4}\left(\varphi_{1}^{*}\right) e^{-(1 / 2) \xi}, & -\xi_{4}<\xi<\infty,
\end{array}
$$




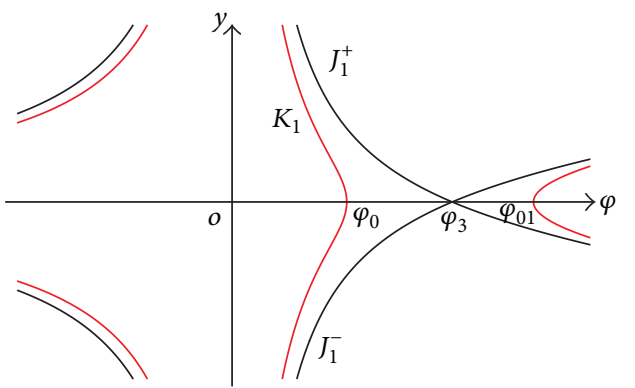

(a)

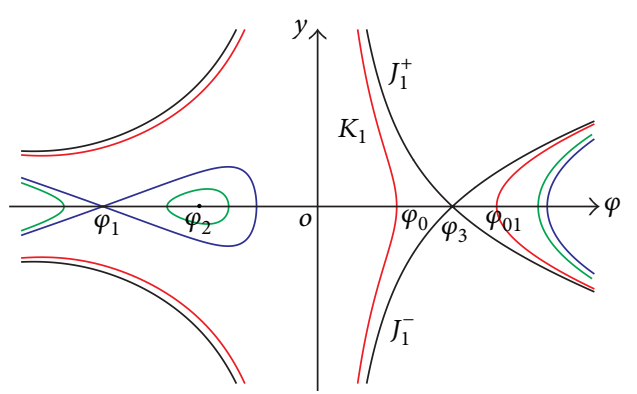

(c)

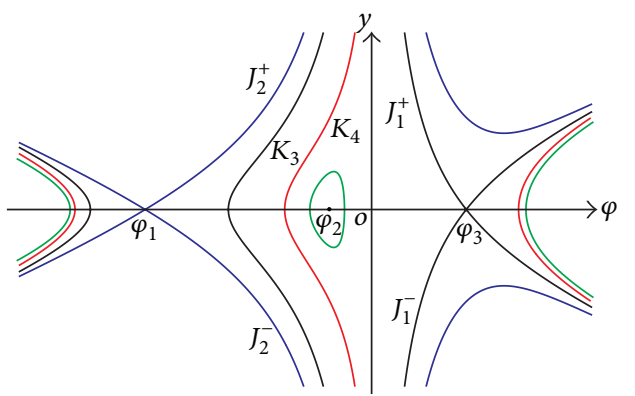

(e)

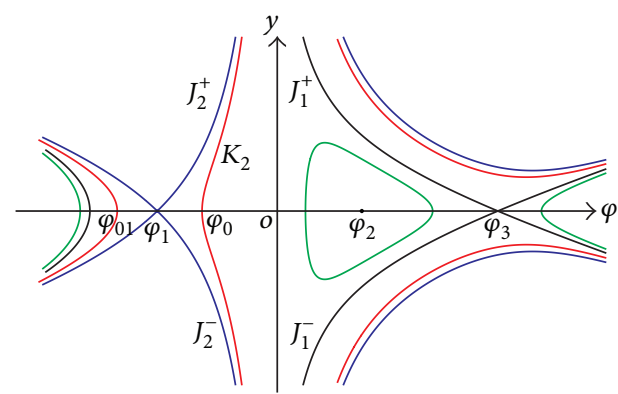

(g)

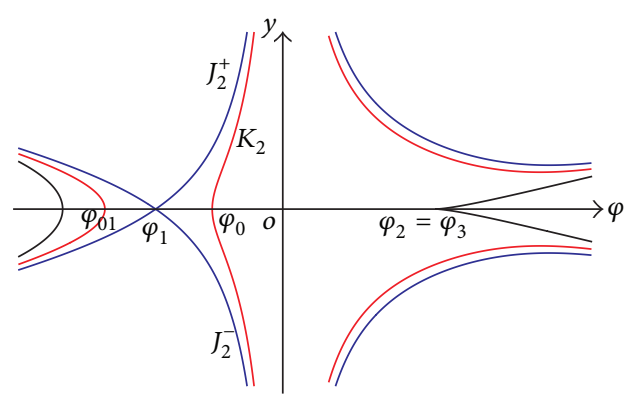

(i)

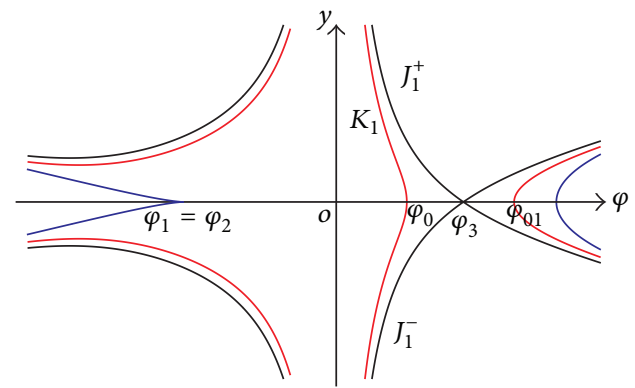

(b)

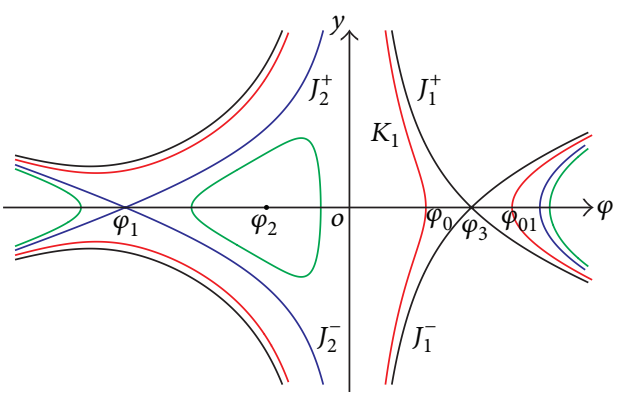

(d)

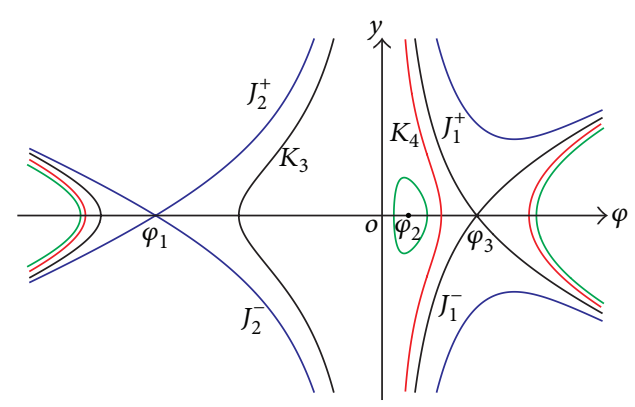

(f)

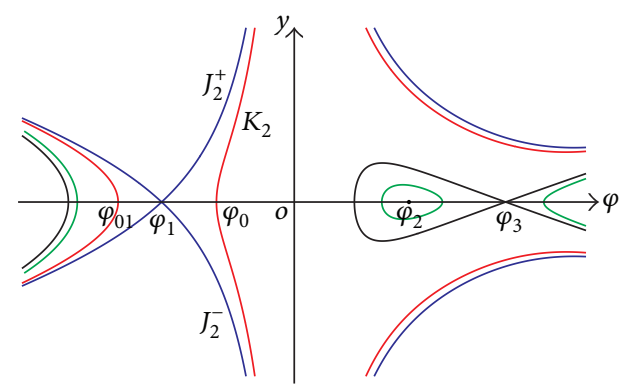

(h)

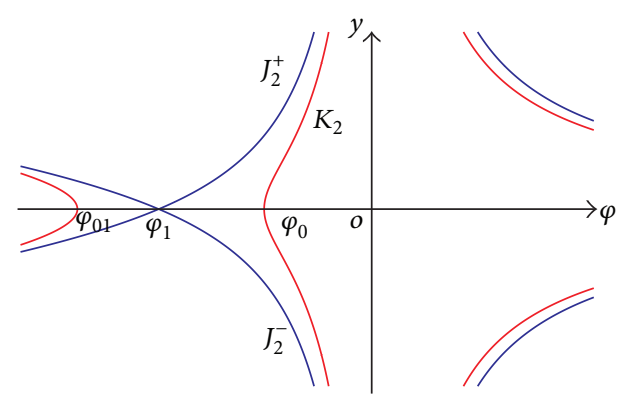

(j)

Figure 1: Continued. 


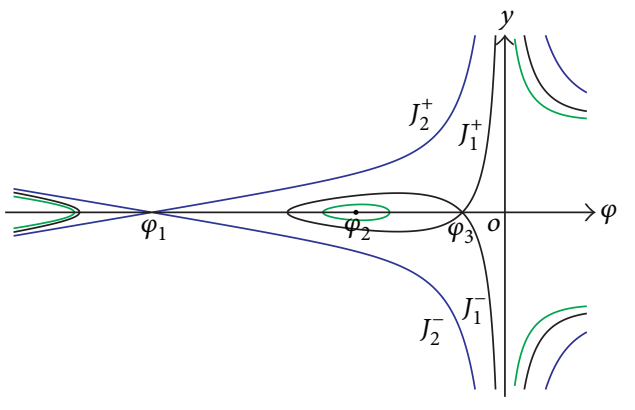

(k)

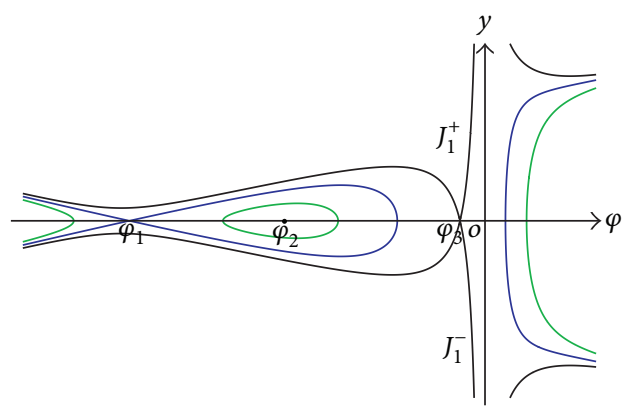

(m)

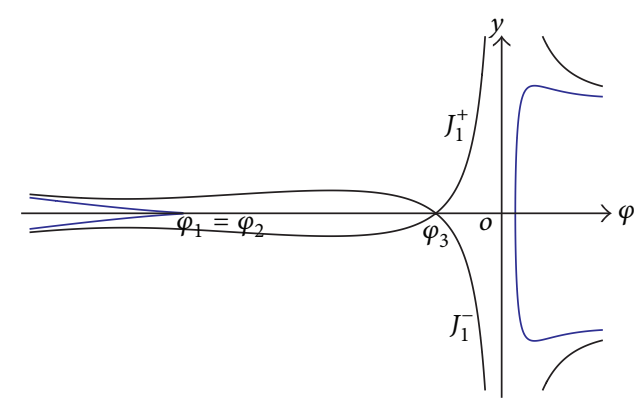

(o)

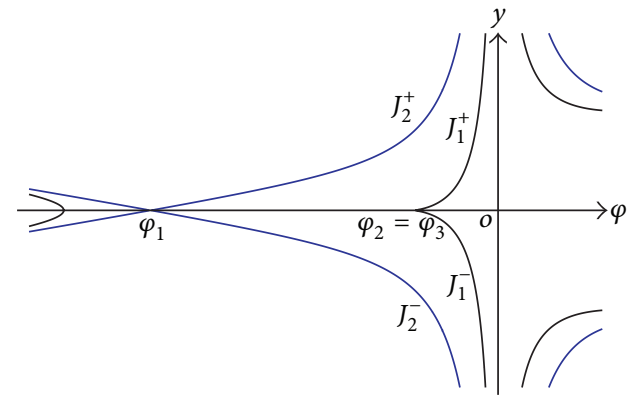

(1)

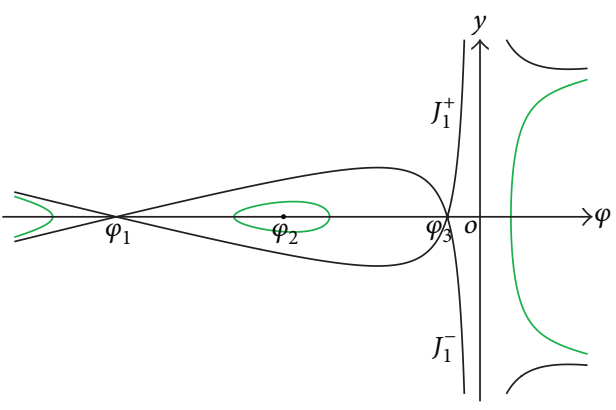

(n)

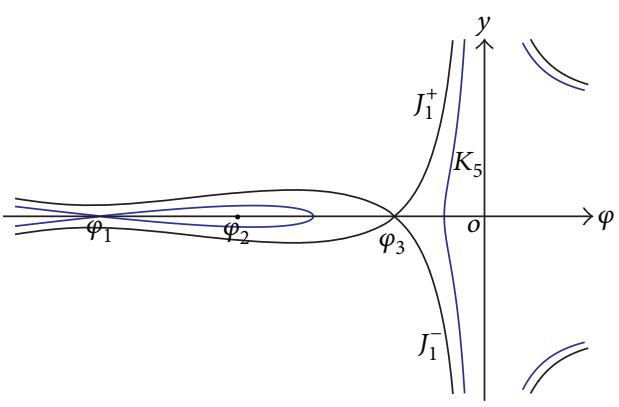

(p)

FIgURE 1: The phase portraits of system (10) in different cases $i(i=1-16)$.

where

$$
\begin{gathered}
\xi_{4}=2 \ln \frac{f_{4}(0)}{f_{4}\left(\varphi_{1}^{*}\right)}, \\
f_{4}(s)=\frac{\left(2 \sqrt{a_{2}} \sqrt{\left(s-\varphi_{3 l}\right)\left(s-\varphi_{3 r}\right)}-b_{2}\left(s-\varphi_{3}\right)+2 a_{2}\right)^{\mu_{2}}}{\left(s-\varphi_{3}\right)^{\mu_{2}}\left(2 \sqrt{\left(s-\varphi_{3 l}\right)\left(s-\varphi_{3 r}\right)}+2 s-\varphi_{3 l}-\varphi_{3 r}\right)} .
\end{gathered}
$$

(5) In Case 12 corresponding to phase portraits in Figure 1(l), we obtain two kink-like wave solutions of implicit expression as follows:

$$
\begin{array}{ll}
f_{5}(\varphi)=f_{5}\left(\varphi_{1}^{*}\right)+\frac{1}{2} \xi, & -\infty<\xi<\xi_{5}, \\
f_{5}(\varphi)=f_{5}\left(\varphi_{1}^{*}\right)-\frac{1}{2} \xi, & -\xi_{5}<\xi<\infty,
\end{array}
$$

where

$$
\begin{gathered}
\xi_{5}=2\left(f_{5}(0)-f_{5}\left(\varphi_{1}^{*}\right)\right), \\
f_{5}(s)=\frac{2 \varphi_{3}}{\varphi_{3}-\varphi_{32}} \sqrt{\frac{s-\varphi_{32}}{s-\varphi_{3}}} \\
-\ln \left(2 \sqrt{\left(s-\varphi_{3}\right)\left(s-\varphi_{32}\right)}+2 s-\varphi_{3}-\varphi_{32}\right), \\
\varphi_{3}=\frac{1}{3}(-2+\sqrt{4-6 \theta}), \quad \varphi_{32}=-\frac{8}{3}-3 \varphi_{3} .
\end{gathered}
$$

(6) In Cases $i(i=13,15,16)$ corresponding to phase portraits in Figures 1(m), 1(o), and 1(p), we obtain two kinklike wave solutions of implicit expression as follows:

$$
\begin{array}{ll}
f_{6}(\varphi)=f_{6}\left(\varphi_{1}^{*}\right) e^{(1 / 2) \xi}, & -\infty<\xi<\xi_{6}, \\
f_{6}(\varphi)=f_{6}\left(\varphi_{1}^{*}\right) e^{-(1 / 2) \xi}, & -\xi_{6}<\xi<\infty,
\end{array}
$$


where

$$
\begin{gathered}
\xi_{6}=2 \ln \frac{f_{6}(0)}{f_{6}\left(\varphi_{1}^{*}\right)} \\
f_{6}(s)=\frac{\left(2 \sqrt{a_{1}} \sqrt{s^{2}+m_{1} s+n_{1}}-b_{1}\left(s-\varphi_{3}\right)+2 a_{1}\right)^{\mu_{1}}}{\left(s-\varphi_{3}\right)^{\mu_{1}}\left(2 \sqrt{s^{2}+m_{1} s+n_{1}}+2 s+m_{1}\right)} .
\end{gathered}
$$

(7) In Case 14 corresponding to phase portrait in Figure 1(n), we obtain two kink-like wave solutions of implicit expression as follows:

$$
\begin{aligned}
& f_{7}(\varphi)=f_{7}\left(\varphi_{1}^{*}\right)+\frac{1}{2} \xi, \quad-\infty<\xi<\xi_{7}, \\
& f_{7}(\varphi)=f_{7}\left(\varphi_{1}^{*}\right)-\frac{1}{2} \xi, \quad-\xi_{7}<\xi<\infty,
\end{aligned}
$$

where

$$
\begin{gathered}
\xi_{7}=2 \ln \frac{f_{7}(0)}{f_{7}\left(\varphi_{1}^{*}\right)}, \\
f_{7}(s)=\frac{1}{\varphi_{3}-\varphi_{1}} \ln \frac{\left(s-\varphi_{1}\right)^{\varphi_{1}}}{\left(s-\varphi_{3}\right)^{\varphi_{3}}} .
\end{gathered}
$$

(8) In Case 4 corresponding to phase portrait in Figure 1(d), we obtain two kink-like wave solutions of implicit expression as follows:

$$
\begin{array}{ll}
f_{8}(\varphi)=f_{8}\left(\varphi_{2}^{*}\right) e^{(1 / 2) \xi}, & -\infty<\xi<\xi_{8}, \\
f_{8}(\varphi)=f_{8}\left(\varphi_{2}^{*}\right) e^{-(1 / 2) \xi}, & -\xi_{8}<\xi<\infty,
\end{array}
$$

where $\varphi_{1}<\varphi_{2}^{*}<0$ and

$$
\begin{gathered}
\xi_{8}=2 \ln \frac{f_{8}(0)}{f_{8}\left(\varphi_{2}^{*}\right)}, \\
f_{8}(s)=\frac{\left(\sqrt{\varphi_{1}^{\circ}-s}+\sqrt{-s}\right)\left(\sqrt{\varphi_{1}\left(s-\varphi_{1}^{\circ}\right)}-\sqrt{\left(\varphi_{1}-\varphi_{1}^{\circ}\right) s}\right)^{\mu_{4}}}{\left(\sqrt{\varphi_{1}^{\circ}-s}-\sqrt{-s}\right)\left(\sqrt{\varphi_{1}\left(s-\varphi_{1}^{\circ}\right)}+\sqrt{\left(\varphi_{1}-\varphi_{1}^{\circ}\right)}\right)^{\mu_{4}}} \\
\mu_{4}=\sqrt{\frac{\varphi_{1}}{\varphi_{1}-\varphi_{1}^{\circ}}}, \quad \varphi_{1}^{\circ}=-\frac{8}{3}-2 \varphi_{1} .
\end{gathered}
$$

(9) In Cases $i(i=5-12)$ corresponding to phase portraits in Figures 1(e)-1(1), we obtain two kink-like wave solutions of implicit expression as follows:

$$
\begin{array}{ll}
f_{9}(\varphi)=f_{9}\left(\varphi_{2}^{*}\right) e^{(1 / 2) \xi}, & -\infty<\xi<\xi_{9}, \\
f_{9}(\varphi)=f_{9}\left(\varphi_{2}^{*}\right) e^{-(1 / 2) \xi}, & -\xi_{9}<\xi<\infty,
\end{array}
$$

where

$$
\begin{gathered}
\xi_{9}=2 \ln \frac{f_{9}(0)}{f_{9}\left(\varphi_{2}^{*}\right)} \\
f_{9}(s)=\frac{\left(2 \sqrt{a_{3}} \sqrt{s^{2}+m_{2} s+n_{2}}+b_{3}\left(s-\varphi_{1}\right)+2 a_{3}\right)^{\mu_{5}}}{\left(s-\varphi_{1}\right)^{\mu_{5}}\left(2 \sqrt{s^{2}+m_{2} s+n_{2}}+2 s+m_{2}\right)} \\
\mu_{5}=\frac{\varphi_{1}}{\sqrt{a_{3}}}, \\
a_{3}=\varphi_{1}^{2}+m_{2} \varphi_{1}+n_{2}, \quad b_{3}=2 \varphi_{1}+m_{2}, \\
m_{2}=\frac{8}{3}+2 \varphi_{1}, \quad n_{2}=4 \theta+\frac{1}{3} \varphi_{1}\left(16+9 \varphi_{1}\right) .
\end{gathered}
$$

4.2. Solutions of Compacton-Like Wave. (1) In Cases $i(i=1-$ $4)$ corresponding to phase portraits in Figures $1(a)-1(d)$ and $\varphi(0)=\varphi_{0},(20)$ becomes

$$
F(\varphi)=\frac{1}{4}\left(\varphi_{0}-\varphi\right)\left(\varphi_{01}-\varphi\right)\left(\varphi-\varphi_{02}\right)\left(\varphi-\bar{\varphi}_{02}\right),
$$

where $\varphi_{0}$ and $\varphi_{01}$ are real roots and $\varphi_{02}$ and $\bar{\varphi}_{02}$ are conjugate complex roots of $F(\varphi)=0$ and $0<\varphi_{0}<\varphi_{3}<\varphi_{01}$. Thus the orbit $K_{1}$ has expressions:

$$
y= \pm \frac{\sqrt{\left(\varphi_{0}-\varphi\right)\left(\varphi_{01}-\varphi\right)\left(\varphi-\varphi_{02}\right)\left(\varphi-\bar{\varphi}_{02}\right)}}{2 \varphi},
$$

where $0<\varphi \leq \varphi_{0}$. By applying transformation $\mathrm{d} \xi=2 \varphi \mathrm{d} v$ to $\mathrm{d} \varphi / \mathrm{d} \xi=y$, we have

$$
\frac{\mathrm{d} \varphi}{\mathrm{d} v}=2 \varphi y .
$$

Substituting (43) into (44) and integrating along $K_{1}$, we get

$$
\varphi=\frac{p \varphi_{01}-q \varphi_{0}-\left(p \varphi_{01}+q \varphi_{0}\right) \mathrm{cn}\left(w, k_{1}\right)}{p-q-(p+q) \mathrm{cn}\left(w, k_{1}\right)}, \quad|w| \leq w_{1},
$$

where

$$
\begin{gathered}
k_{1}=\sqrt{\frac{(p+q)^{2}-\left(\varphi_{0}-\varphi_{01}\right)^{2}}{4 p q}}, \\
p=\sqrt{\left(A-\varphi_{01}\right)^{2}+B^{2}}, \quad q=\sqrt{\left(A-\varphi_{0}\right)^{2}+B^{2}}, \\
A=\frac{\varphi_{02}-\bar{\varphi}_{02}}{2}, \quad B=-\frac{\left(\varphi_{02}-\bar{\varphi}_{02}\right)^{2}}{4}, \\
w=\sqrt{p q} v, \quad w_{1}=\mathrm{cn}^{-1}\left(\frac{p \varphi_{01}-q \varphi_{0}}{p \varphi_{01}+q \varphi_{0}}, k_{1}\right) .
\end{gathered}
$$




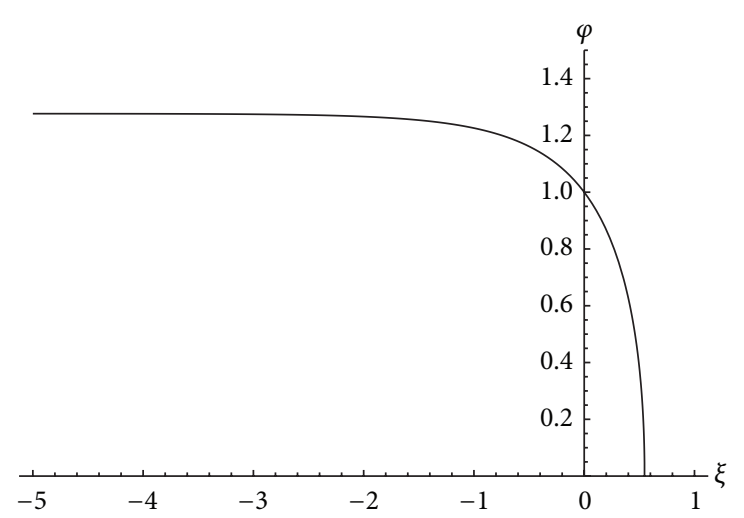

(a)

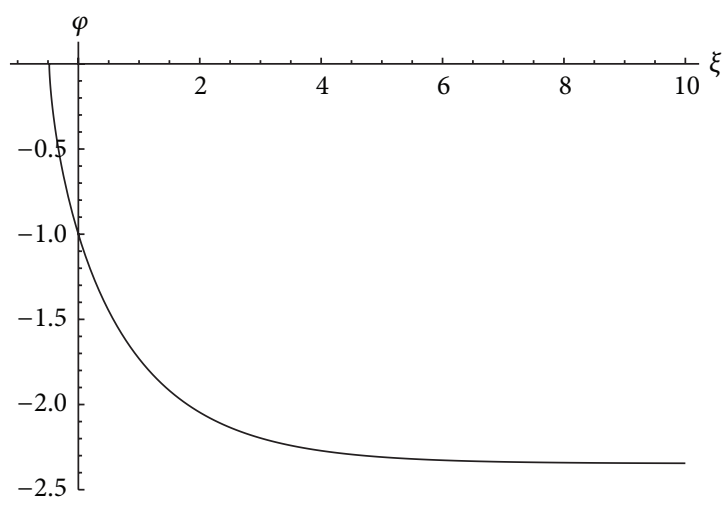

(c)

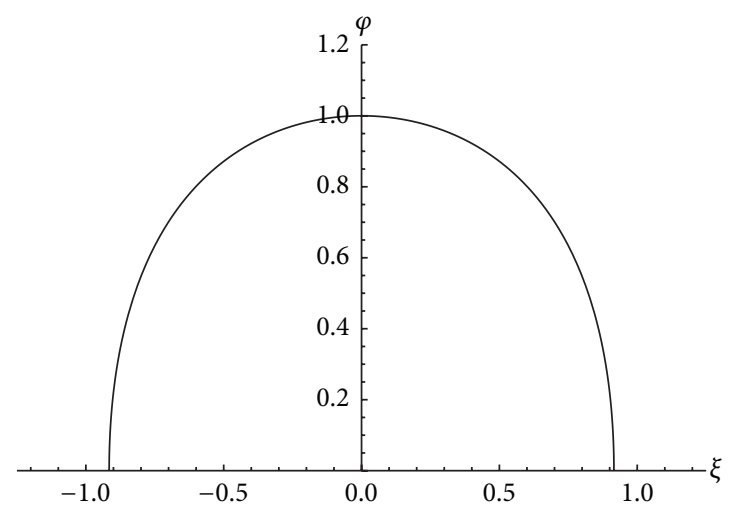

(e)

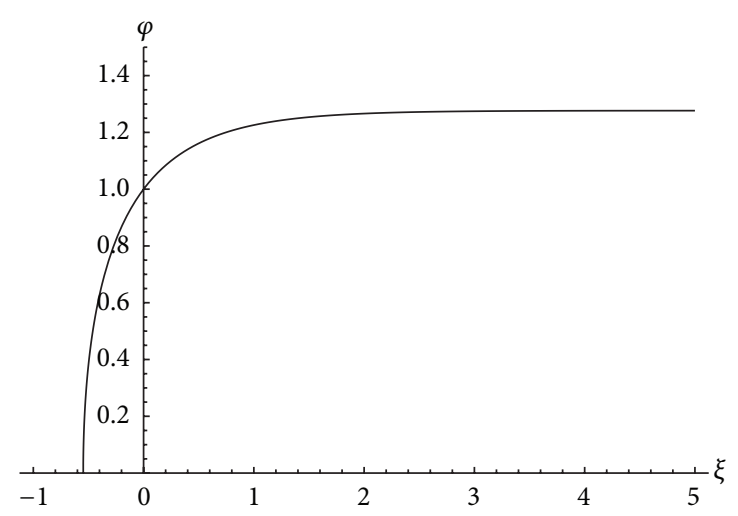

(b)

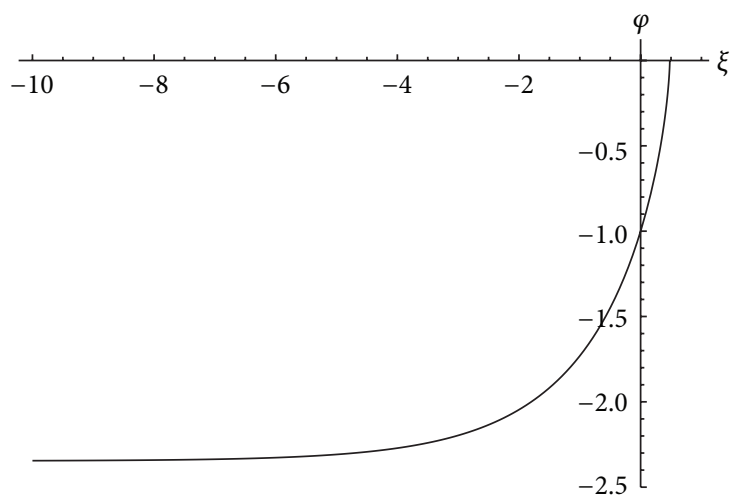

(d)

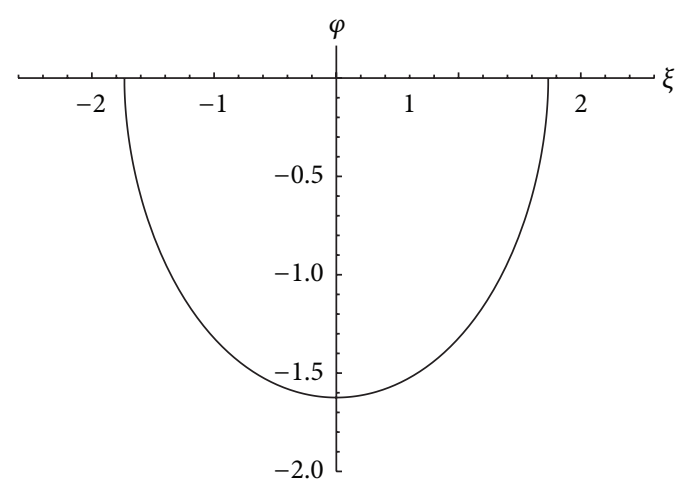

(f)

FIGURE 2: The simulations of integral curves of (8) when $\theta=-1, g=-1.39361$ (corresponding to Example 1), and $g=-0.696804$ (corresponding to Example 2). (a) $\varphi(0)=1$ and $\varphi^{\prime}(0)=-0.5160826789900144$. (b) $\varphi(0)=1$ and $\varphi^{\prime}(0)=0.5160826789900144$. (c) $\varphi(0)=-1$ and $\varphi^{\prime}(0)=-1.1707040231039854$. (d) $\varphi(0)=-1$ and $\varphi^{\prime}(0)=1.1707040231039854$. (e) $\varphi(0)=1$ and $\varphi^{\prime}(0)=0$. (f) $\varphi(0)=-1.624378546433569$ and $\varphi^{\prime}(0)=0$. get

Substituting (45) into $\mathrm{d} \xi=2 \varphi \mathrm{d} v$ and integrating once, we where

$$
\begin{aligned}
\xi=\frac{2}{\sqrt{p q}}( & \frac{p \varphi_{01}+q \varphi_{0}}{p+q} w+\frac{(p-q)\left(\varphi_{0}-\varphi_{01}\right)}{2(p+q)} \\
& \left.\times\left(\Pi\left(\sin ^{-1}\left(\operatorname{sn}\left(w, k_{1}\right)\right), \frac{\alpha_{1}^{2}}{\alpha_{1}^{2}-1}, k_{1}\right)-\alpha_{1} \beta\right)\right),
\end{aligned}
$$

$$
\begin{gathered}
\alpha_{1}=\frac{p+q}{q-p}, \\
\beta=\frac{1}{2} \sqrt{\frac{\alpha_{1}^{2}-1}{k_{1}^{2}+\left(k_{1}^{\prime} \alpha_{1}\right)^{2}}}
\end{gathered}
$$




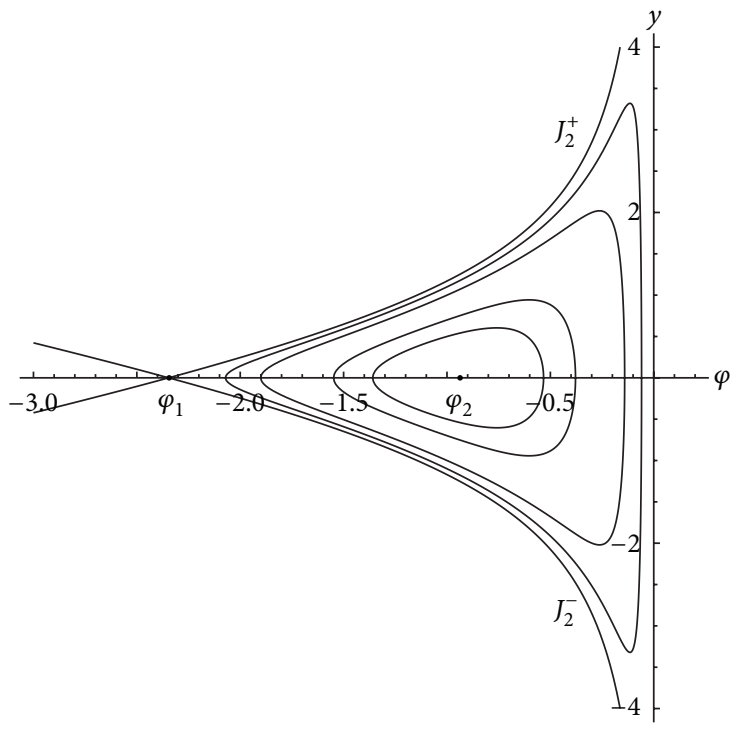

(a)

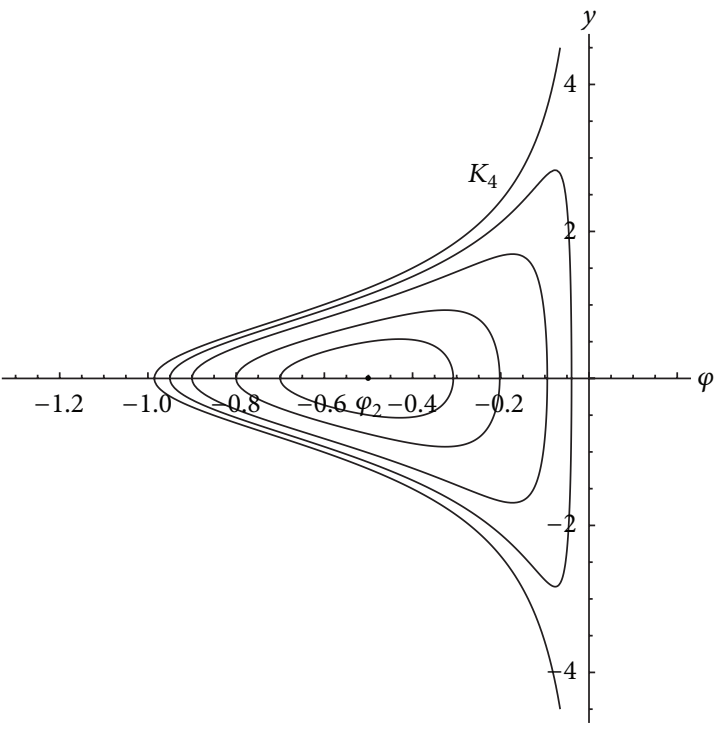

(b)

FIgURE 3: The periodic orbits surrounding the center point $\left(\varphi_{2}, 0\right)$ and their boundaries, where (a) for Case 4 and (b) for Case 5.

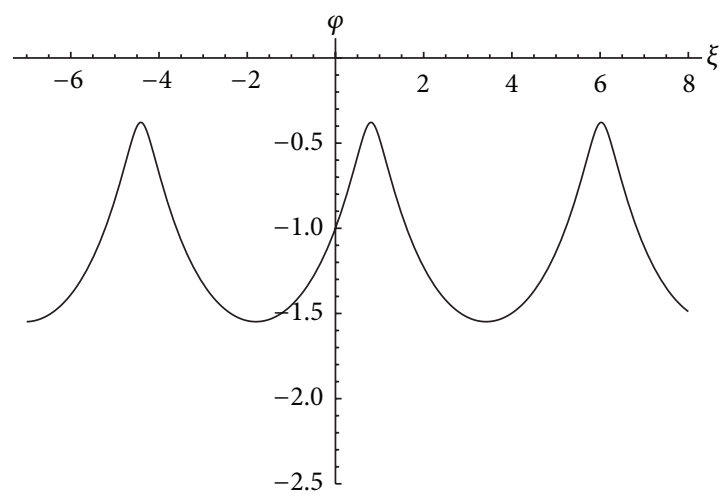

(a)

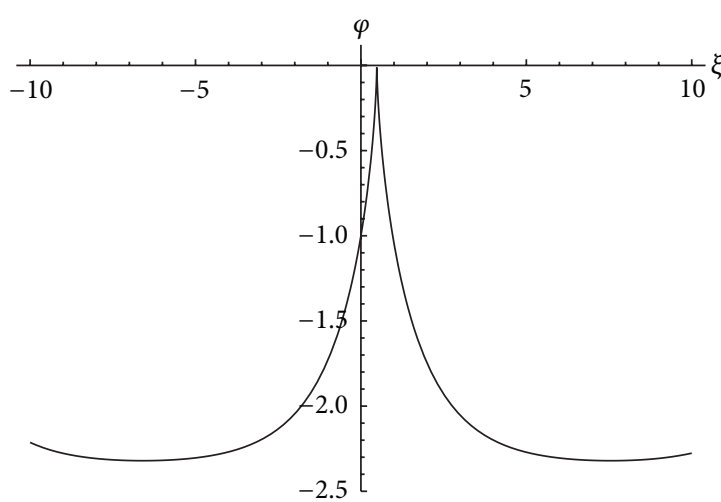

(c)

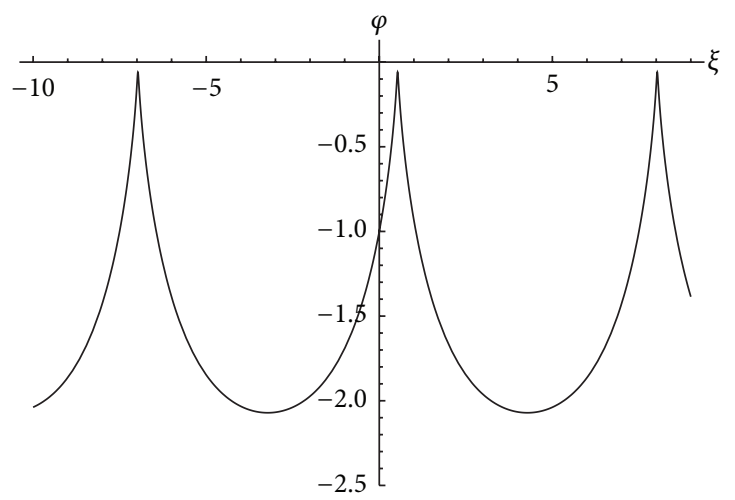

(b)

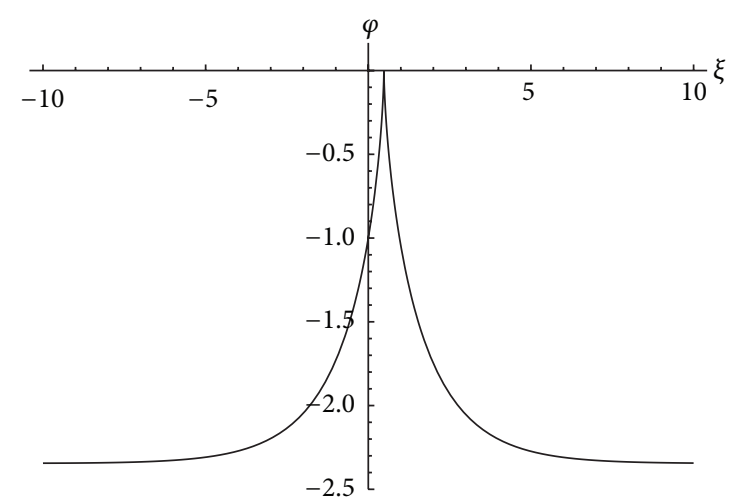

(d)

Figure 4: The simulations of periodic integral curves of (8) for Case 4, where the initial values $\left(\varphi(0), \varphi^{\prime}(0)\right)=$ $(-1,0.7),(-1,1.1),(-1,1.17),(-1,1.170704)$. 


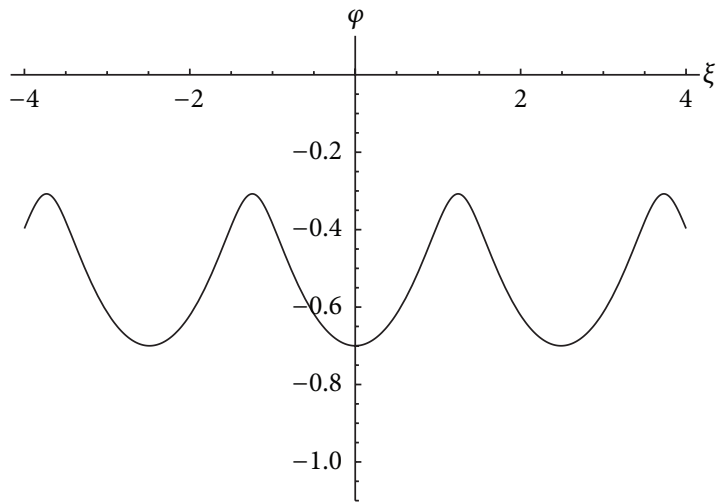

(a)

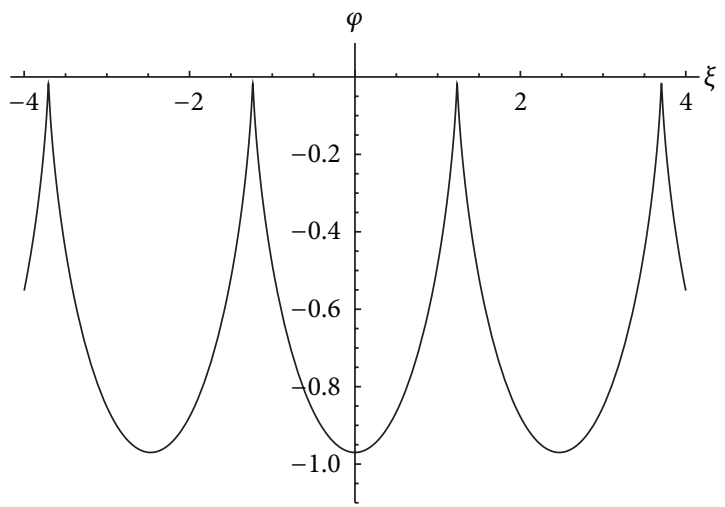

(c)

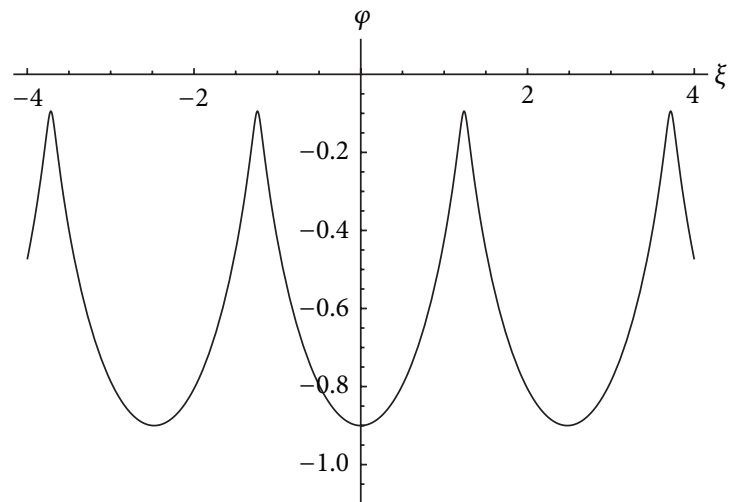

(b)

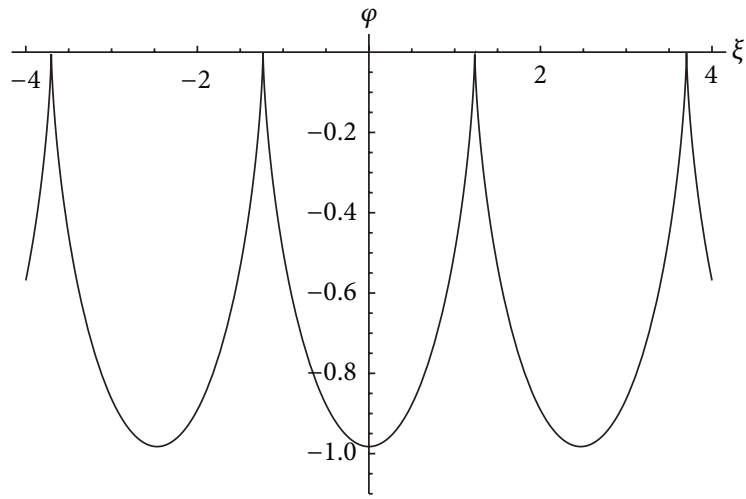

(d)

Figure 5: The simulations of periodic integral curves of (8) for Case 5 , where the initial values $\left(\varphi(0), \varphi^{\prime}(0)\right)=(-0.7,0),(-0.9,0),(-0.98,0)$, $(-0.9854465,0)$.

$$
\begin{gathered}
\times \ln \frac{\sqrt{k_{1}^{2}+\left(k_{1}^{\prime} \alpha_{1}\right)^{2}} \operatorname{dn}\left(w, k_{1}\right)+\sqrt{\alpha_{1}^{2}-1} \operatorname{sn}\left(w, k_{1}\right)}{\sqrt{k_{1}^{2}+\left(k_{1}^{\prime} \alpha_{1}\right)^{2}} \operatorname{dn}\left(w, k_{1}\right)-\sqrt{\alpha_{1}^{2}-1} \operatorname{sn}\left(w, k_{1}\right)}, \\
k_{1}^{\prime}=\sqrt{1-k_{1}^{2}} .
\end{gathered}
$$

Thus we obtain a compacton-like wave solution of parametric expression as follows:

$$
\begin{gathered}
\varphi=\frac{p \varphi_{01}-q \varphi_{0}-\left(p \varphi_{01}+q \varphi_{0}\right) \mathrm{cn}\left(w, k_{1}\right)}{p-q-(p+q) \operatorname{cn}\left(w, k_{1}\right)}, \\
\xi=\frac{2}{\sqrt{p q}}\left(\frac{p \varphi_{01}+q \varphi_{0}}{p+q} w+\frac{(p-q)\left(\varphi_{0}-\varphi_{01}\right)}{2(p+q)}\right. \\
\times\left(\Pi\left(\sin ^{-1}\left(\operatorname{sn}\left(w, k_{1}\right)\right), \frac{\alpha_{1}^{2}}{\alpha_{1}^{2}-1}, k_{1}\right)\right. \\
\left.\left.-\alpha_{1} \beta\right)\right),
\end{gathered}
$$

where $w$ is a parameter variable and $-w_{1} \leq w \leq w_{1}, 0<\varphi \leq$ $\varphi_{0}$.
The derivations of other compacton-like solutions are similar to the above case, so we omit the details and only list the results.

(2) In Cases $i(i=7-10)$ corresponding to phase portraits in Figures $1(\mathrm{~g})-1(\mathrm{j})$, we obtain a compacton-like wave solution of parametric expression as follows:

$$
\begin{gathered}
\varphi=\frac{p \varphi_{0}-q \varphi_{01}+\left(p \varphi_{0}+q \varphi_{01}\right) \mathrm{cn}\left(w, k_{1}\right)}{p-q+(p+q) \mathrm{cn}\left(w, k_{1}\right)}, \\
\xi=\frac{2}{\sqrt{p q}}\left(\frac{p \varphi_{0}+q \varphi_{01}}{p+q} w-\frac{(p-q)\left(\varphi_{0}-\varphi_{01}\right)}{2(p+q)}\right. \\
\times\left(\Pi\left(\sin ^{-1}\left(\operatorname{sn}\left(w, k_{1}\right)\right), \frac{\alpha_{1}^{2}}{\alpha_{1}^{2}-1}, k_{1}\right)\right. \\
\left.\left.+\alpha_{1} \beta\right)\right),
\end{gathered}
$$

where $w$ is a parameter variable, $-w_{2} \leq w \leq w_{2}$, and $w_{2}=$ $\mathrm{cn}^{-1}\left(\left(\left(p \varphi_{0}-q \varphi_{01}\right) /\left(p \varphi_{0}+q \varphi_{01}\right)\right) \cdot k_{1}\right), \varphi_{0} \leq \varphi<0$,

(3) In Cases $i(i=5,6)$ corresponding to phase portraits in Figures 1(e) and 1(f), we obtain a compacton-like wave solution of implicit expression as follows:

$$
f_{2}(\varphi)=f_{2}\left(\varphi_{3 r}\right) e^{-(1 / 2)|\xi|}, \quad|\xi| \leq \xi_{10},
$$


where

$$
\xi_{10}=2 \ln \frac{f_{2}\left(\varphi_{3 r}\right)}{f_{2}(0)}
$$

(4) In Case 5 corresponding to phase portraits in Figure 1(e), we obtain a compacton-like wave solution of implicit expression as follows:

$$
\begin{aligned}
4 \sqrt{\frac{-\varphi_{0 l}^{\circ}}{\varphi_{0 r}^{\circ}-\varphi_{0}^{\circ}}}\left(\operatorname{sn}^{-1}\left(\sqrt{\frac{\varphi_{0 l}^{\circ}\left(\varphi-\varphi_{0}^{\circ}\right)}{\varphi_{0}^{\circ}\left(\varphi-\varphi_{0 l}^{\circ}\right)}}, k_{2}\right)-\frac{\varphi_{0 l}^{\circ}-\varphi_{0}^{\circ}}{\varphi_{0 l}^{\circ}}\right. \\
\left.\times \Pi\left(\sin ^{-1}\left(\sqrt{\frac{\varphi_{0 l}^{\circ}\left(\varphi-\varphi_{0}^{\circ}\right)}{\varphi_{0}^{\circ}\left(\varphi-\varphi_{0 l}^{\circ}\right)}}\right), \alpha_{2}^{2}, k_{2}\right)\right)
\end{aligned}
$$$$
=|\xi|, \quad|\xi| \leq \xi_{11}
$$

where $\varphi_{0}^{\circ}$ is a root of equation $F(\varphi)=0$ and

$$
\begin{gathered}
\xi_{11}=4 \sqrt{\frac{-\varphi_{0 l}^{\circ}}{\varphi_{0 r}^{\circ}-\varphi_{0}^{\circ}}}\left(\operatorname{sn}^{-1}\left(1, k_{2}\right)-\frac{\varphi_{0 l}^{\circ}-\varphi_{0}^{\circ}}{\varphi_{0 l}^{\circ}} \Pi\left(\frac{\pi}{2}, \alpha_{2}^{2}, k_{2}\right)\right), \\
\alpha_{2}=\sqrt{\frac{\varphi_{0}^{\circ}}{\varphi_{0 l}^{\circ}}}, \quad k_{2}=\sqrt{\frac{\left(\varphi_{0 r}^{\circ}-\varphi_{0 l}^{\circ}\right) \varphi_{0}^{\circ}}{\left(\varphi_{0 r}^{\circ}-\varphi_{0}^{\circ}\right) \varphi_{0 l}^{\circ}}} \\
\varphi_{0 l}^{\circ}=\frac{1}{6}\left(-8-3 \varphi_{0}^{\circ}-\sqrt{-144 \theta-\left(8+3 \varphi_{0}^{\circ}\right)\left(-8+9 \varphi_{0}^{\circ}\right)}\right), \\
\varphi_{0 r}^{\circ}=\frac{1}{6}\left(-8-3 \varphi_{0}^{\circ}+\sqrt{-144 \theta-\left(8+3 \varphi_{0}^{\circ}\right)\left(-8+9 \varphi_{0}^{\circ}\right)}\right) .
\end{gathered}
$$

(5) In Case 6 corresponding to phase portraits in Figure 1(f), we obtain a compacton-like wave solution of implicit expression as follows:

$$
\begin{aligned}
& 4 \sqrt{\frac{\varphi_{0 r}^{\circ}}{\varphi_{0}^{\circ}-\varphi_{0 l}^{\circ}}}\left(\operatorname{sn}^{-1}\left(\sqrt{\frac{\varphi_{0 r}^{\circ}\left(\varphi_{0}^{\circ}-\varphi\right)}{\varphi_{0}^{\circ}\left(\varphi_{0 r}^{\circ}-\varphi\right)}}, k_{3}\right)+\frac{\varphi_{0}^{\circ}-\varphi_{0 r}^{\circ}}{\varphi_{0 r}^{\circ}}\right. \\
& \left.\quad \times \Pi\left(\sin ^{-1}\left(\sqrt{\frac{\varphi_{0 r}^{\circ}\left(\varphi_{0}^{\circ}-\varphi\right)}{\varphi_{0}^{\circ}\left(\varphi_{0 r}^{\circ}-\varphi\right)}}\right), \alpha_{3}^{2}, k_{3}\right)\right) \\
& =|\xi|, \quad|\xi| \leq \xi_{12},
\end{aligned}
$$

where

$$
\begin{gathered}
\xi_{12}=4 \sqrt{\frac{\varphi_{0 r}^{\circ}}{\varphi_{0}^{\circ}-\varphi_{0 l}^{\circ}}}\left(\operatorname{sn}^{-1}\left(1, k_{3}\right)+\frac{\varphi_{0}^{\circ}-\varphi_{0 r}^{\circ}}{\varphi_{0 r}^{\circ}} \Pi\left(\frac{\pi}{2}, \alpha_{3}^{2}, k_{3}\right)\right), \\
\alpha_{3}=\sqrt{\frac{\varphi_{0}^{\circ}}{\varphi_{0 r}^{\circ}}}, \quad k_{3}=\sqrt{\frac{\left(\varphi_{0 r}^{\circ}-\varphi_{0 l}^{\circ}\right) \varphi_{0}^{\circ}}{\left(\varphi_{0}^{\circ}-\varphi_{0 l}^{\circ}\right) \varphi_{0 r}^{\circ}}}
\end{gathered}
$$

(6) In Case 16 corresponding to phase portraits in Figure 1(p), we obtain a compacton-like wave solution of implicit expression as follows:

$$
f_{10}(\varphi)=f_{10}\left(\varphi_{1 r}\right) e^{(1 / 2)|\xi|}, \quad|\xi| \leq \xi_{13}
$$

where

$$
\begin{gathered}
\xi_{13}=2 \ln \frac{f_{10}(0)}{f_{10}\left(\varphi_{1 r}\right)}, \\
f_{10}(s)=\frac{\left(2 \sqrt{a_{4}} \sqrt{\left(s-\varphi_{1 l}\right)\left(s-\varphi_{1 r}\right)}+b_{4}\left(s-\varphi_{1}\right)+2 a_{4}\right)^{\mu_{6}}}{\left(s-\varphi_{1}\right)^{\mu_{6}}\left(2 \sqrt{\left(s-\varphi_{1 l}\right)\left(s-\varphi_{1 r}\right)}+2 s-\varphi_{1 l}-\varphi_{1 r}\right)}, \\
\mu_{6}=\frac{\varphi_{1}}{\sqrt{a_{4}}}, \\
a_{4}=\left(\varphi_{1}-\varphi_{1 l}\right)\left(\varphi_{1}-\varphi_{1 r}\right), \quad b_{4}=2 \varphi_{1}-\varphi_{1 l}+\varphi_{1 r}, \\
\varphi_{1 l}=\frac{1}{3}\left(-4-3 \varphi_{1}-\sqrt{2} \sqrt{8-18 \theta-3 \varphi_{1}\left(4+3 \varphi_{1}\right)}\right), \\
\varphi_{1 r}=\frac{1}{3}\left(-4-3 \varphi_{1}+\sqrt{2} \sqrt{8-18 \theta-3 \varphi_{1}\left(4+3 \varphi_{1}\right)}\right) .
\end{gathered}
$$

\section{Conclusion}

In this paper, we have found two types of bounded traveling wave solutions for a two-component Fornberg-Whitham equation (see (4)), that is, the compacton-like wave and kinklike wave solutions. Their planar graphs of these solutions are simulated by using software Mathematica (see Figure 2). Meanwhile we reveal two kinds of new phenomena; that is, the periodic wave solution can become the kink-like wave or compacton-like wave solution under some conditions, respectively (see Figures 4 and 5). In different Cases $i(i=1-$ 16), the exact implicit or parameter expressions of kink-like wave and compacton-like wave solutions are given.

\section{Conflict of Interests}

The authors declare that there is no conflict of interests regarding the publication of this paper.

\section{Acknowledgment}

This paper is supported by the National Natural Science Foundation (no. 11361069), and Science Foundation of Shaoguan University (no. 201320501).

\section{References}

[1] G. B. Whitham, "Variational methods and applications to water wave," Proceedings of the Royal Society A, vol. 299, no. 1456, pp. 6-25, 1967.

[2] B. Fornberg and G. B. Whitham, "A numerical and theoretical study of certain nonlinear wave phenomena," Philosophical Transactions of the Royal Society of London A, vol. 289, no. 1361, pp. 373-404, 1978. 
[3] J. Zhou and L. Tian, "A type of bounded traveling wave solutions for the Fornberg-Whitham equation," Journal of Mathematical Analysis and Applications, vol. 346, no. 1, pp. 255-261, 2008.

[4] J. Zhou and L. Tian, "Solitons, peakons and periodic cusp wave solutions for the Fornberg-Whitham equation," Nonlinear Analysis: Real World Applications, vol. 11, no. 1, pp. 356-363, 2010.

[5] J. Zhou and L. Tian, "Periodic and solitary wave solutions to the Fornberg-Whitham equation," Mathematical Problems in Engineering, vol. 2009, Article ID 507815, 10 pages, 2009.

[6] C. Feng and C. Wu, "The classification of all single traveling wave solutions to Fornberg-Whitham equation," International Journal of Nonlinear Science, vol. 7, no. 3, pp. 353-359, 2009.

[7] A. Chen, J. Li, X. Deng, and W. Huang, "Travelling wave solutions of the Fornberg-Whitham equation," Applied Mathematics and Computation, vol. 215, no. 8, pp. 3068-3075, 2009.

[8] B. He, Q. Meng, and S. Li, "Explicit peakon and solitary wave solutions for the modified Fornberg-Whitham equation," Applied Mathematics and Computation, vol. 217, no. 5, pp. 19761982, 2010.

[9] B. Jiang and Q. Bi, "Smooth and non-smooth traveling wave solutions of the Fornberg-Whitham equation with linear dispersion term," Applied Mathematics and Computation, vol. 216, no. 7, pp. 2155-2162, 2010.

[10] X. Fan, S. Yang, J. Yin, and L. Tian, "Bifurcations of traveling wave solutions for a two-component Fornberg-Whitham equation," Communications in Nonlinear Science and Numerical Simulation, vol. 16, no. 10, pp. 3956-3963, 2011.

[11] Z. Wen, "Extension on bifurcations of traveling wave solutions for a two-component Fornberg-Whitham equation," Abstract and Applied Analysis, vol. 2012, Article ID 704931, 15 pages, 2012.

[12] P. Rosenau and J. M. Hyman, "Compactons: solitons with finite wavelength," Physical Review Letters, vol. 70, no. 5, pp. 564-567, 1993.

[13] P. Rosenau, "Compact and noncompact dispersive patterns," Physics Letters A, vol. 275, no. 3, pp. 193-203, 2000.

[14] Z. Liu, Q. Li, and Q. Lin, "New bounded traveling waves of Camassa-Holm equation," International Journal of Bifurcation and Chaos, vol. 14, no. 10, pp. 3541-3556, 2004.

[15] Z. Liu and Y. Long, "Generalized kink waves in a general compressible hyperelastic rod," International Journal of Bifurcation and Chaos, vol. 15, no. 8, pp. 2671-2679, 2005.

[16] Z.-R. Liu and Y. Long, "Compacton-like wave and kinklike wave of GCH equation," Nonlinear Analysis: Real World Applications, vol. 8, no. 1, pp. 136-155, 2007.

[17] S. Xie and L. Wang, "Compacton and generalized kink wave solutions of the CH-DP equation," Applied Mathematics and Computation, vol. 215, no. 11, pp. 4028-4039, 2010.

[18] S. Xie, Y. Zhang, and J. He, "Two types of bounded travelingwave solutions of a two-component Camassa-Holm equation," Applied Mathematics and Computation, vol. 219, no. 20, pp. 10271-10282, 2013.

[19] G.-T. Liu and T.-Y. Fan, "New applications of developed Jacobi elliptic function expansion methods," Physics Letters A, vol. 345, no. 1-3, pp. 161-166, 2005.

[20] S. Liu, Z. Fu, S. Liu, and Q. Zhao, "Jacobi elliptic function expansion method and periodic wave solutions of nonlinear wave equations," Physics Letters A, vol. 289, no. 1-2, pp. 69-74, 2001.

[21] M. A. Abdou, "The extended F-expansion method and its application for a class of nonlinear evolution equations," Chaos, Solitons \& Fractals, vol. 31, no. 1, pp. 95-104, 2007.
[22] M. Wang and X. Li, "Extended F-expansion method and periodic wave solutions for the generalized Zakharov equations," Physics Letters A, vol. 343, no. 1-3, pp. 48-54, 2005.

[23] M. Wang, X. Li, and J. Zhang, “The $\frac{G^{\prime}}{G}$-expansion method and travelling wave solutions of nonlinear evolution equations in mathematical physics," Physics Letters A, vol. 372, no. 4, pp. 417423, 2008.

[24] M. Song and Y. Ge, "Application of the $\frac{G^{\prime}}{G}$-expansionmethod to $(3+1)$-dimensional nonlinear evolution equations," Computers and Mathematics with Applications, vol. 60, no. 5, pp. 1220-1227, 2010.

[25] J. Li and Z. Liu, "Smooth and non-smooth traveling waves in a nonlinearly dispersive equation," Applied Mathematical Modelling, vol. 25, no. 1, pp. 41-56, 2000.

[26] Z. Liu and C. Yang, "The application of bifurcation method to a higher-order KdV equation," Journal of Mathematical Analysis and Applications, vol. 275, no. 1, pp. 1-12, 2002.

[27] R. Liu, "Some new results on explicit traveling wave solutions of $K(m, n)$ equation," Discrete and Continuous Dynamical Systems $B$, vol. 13, no. 3, pp. 633-646, 2010.

[28] Z. Liu and Y. Liang, "The explicit nonlinear wave solutions and their bifurcations of the generalized camassaholm equation," International Journal of Bifurcation and Chaos, vol. 21, no. 11, pp. 3119-3136, 2011. 


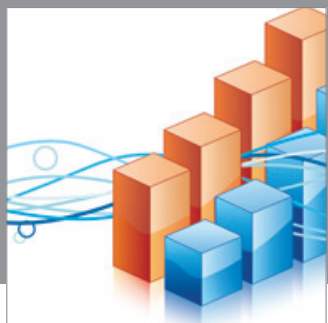

Advances in

Operations Research

mansans

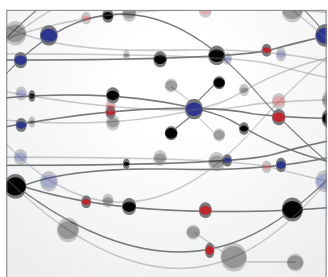

The Scientific World Journal
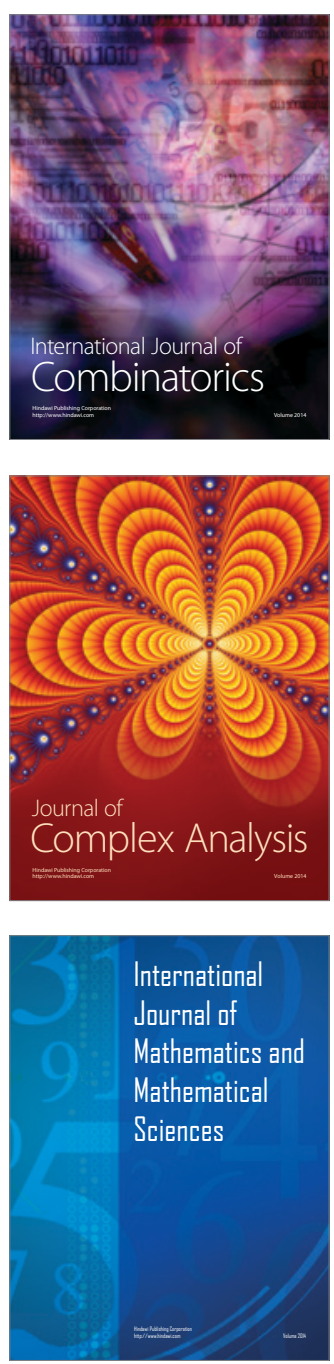
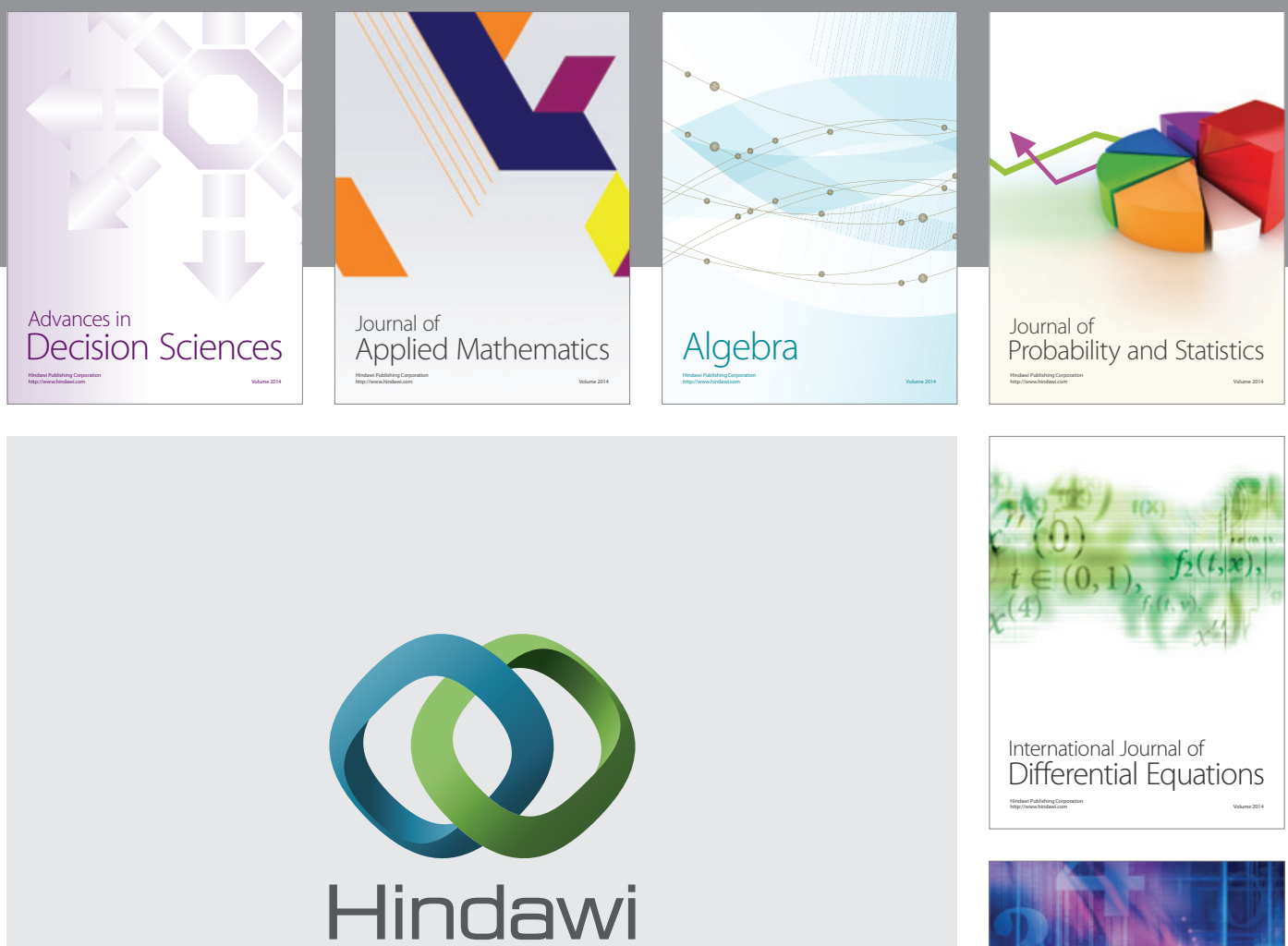

Submit your manuscripts at http://www.hindawi.com
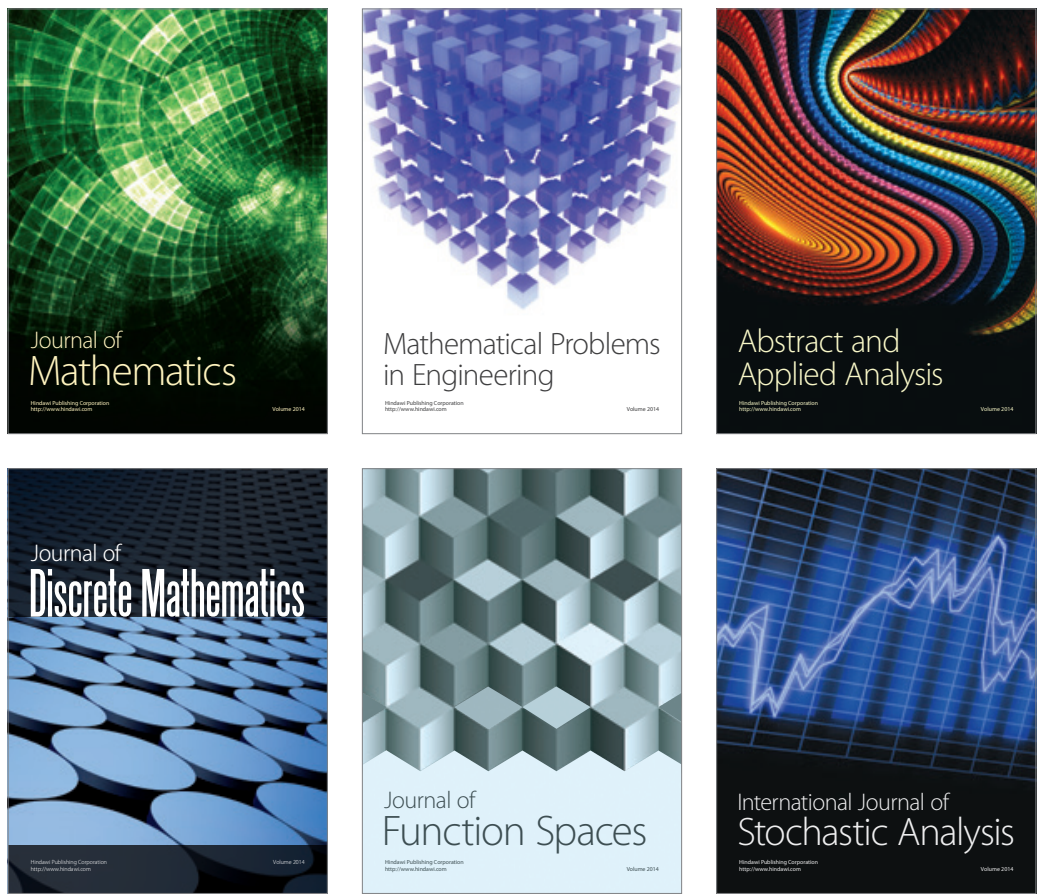

Journal of

Function Spaces

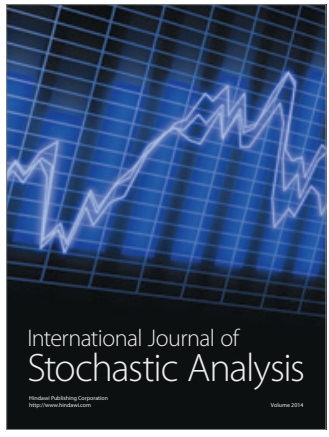

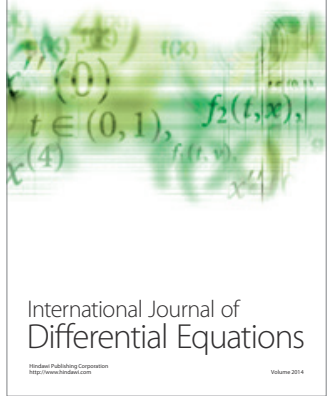
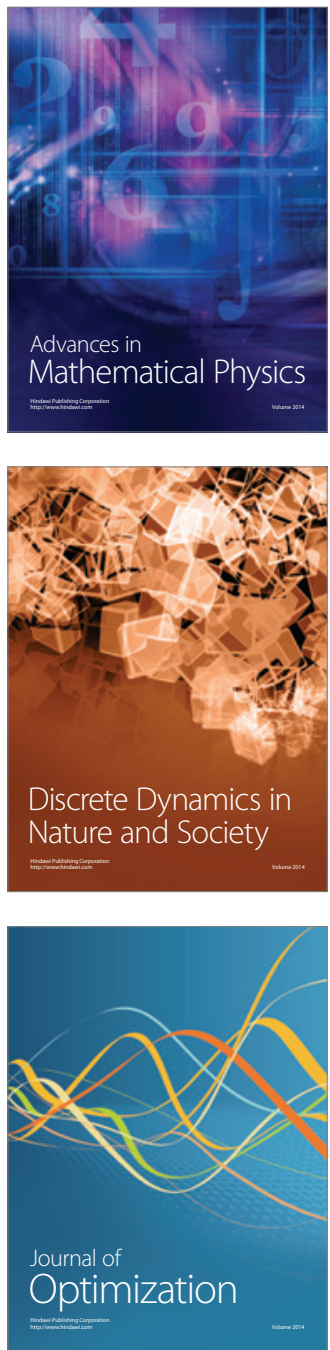